\title{
Preference of 4-Exo Ring Closure in Copper-Catalyzed Intramolecular Coupling of Vinyl Bromides with Alcohols
}

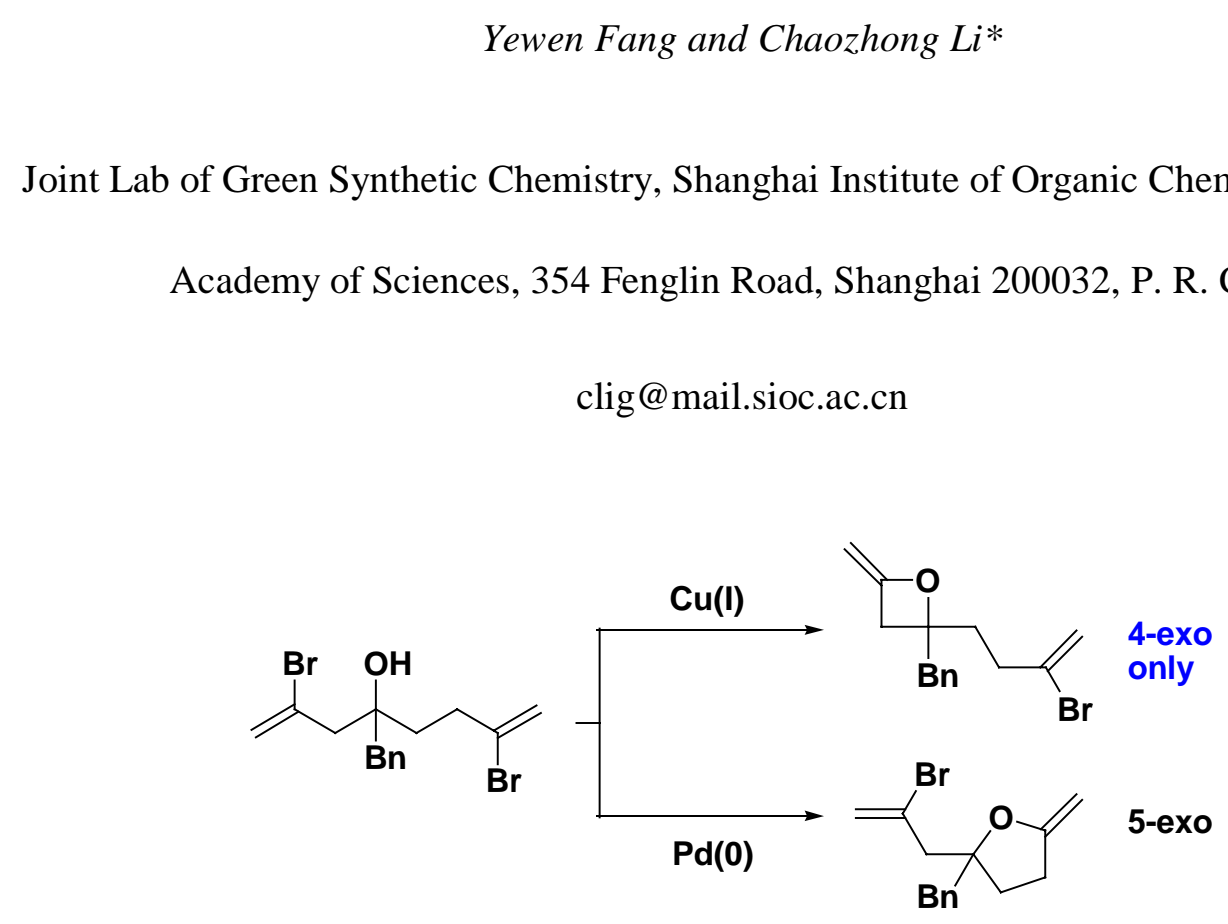

Supporting Information

\section{Table of Content}

1. Synthesis and characterizations of substrates $\mathbf{1}$ and $\mathbf{5}$.

$\mathrm{S} 2-\mathrm{S} 12$

2. Typical procedures for $\mathrm{Cu}$-catalyzed and $\mathrm{Pd}$-catalyzed $\mathrm{O}$-vinylation.

3. Characterizations of products $\mathbf{2 , 6 , 8}$ and 9.

$\mathrm{S} 13-\mathrm{S} 22$

4. References for known compounds.

S22

5. COSY spectra of $\mathbf{6 a}, \mathbf{6 b}$ and NOESY spectra of $\mathbf{2 f}, \mathbf{6} \mathbf{b}$.

$\mathrm{S} 23-\mathrm{S} 26$ 


\section{Synthesis and characterizations of substrates 1 and 5 .}

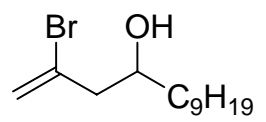

$1 \mathrm{a}$

Substrate 1a was prepared by the Barbier reaction of 2,3-dibromoprop-1-ene with decanal. ${ }^{1}$ Tin powder (1.49 g, $12.5 \mathrm{mmol})$ was added into the mixture of 2,3-dibromoprop-1-ene (5.00 g, $25.0 \mathrm{mmol}), \mathrm{H}_{2} \mathrm{O}(9 \mathrm{~mL})$ and $\mathrm{Et}_{2} \mathrm{O}(9 \mathrm{~mL})$ at room temperature. Three drops of aqueous hydrobromic acid (1 M) was then added. Decanal $(1.30 \mathrm{~g}, 8.33 \mathrm{mmol})$ was added in one portion and the mixture was stirred at room temperature overnight. The resulting mixture was filtered and the precipitate was washed with ether $(20 \mathrm{~mL})$. The two layers of the filtrate were separated and the aqueous phase was extracted with ether $(3 \times 20 \mathrm{~mL})$. The combined organic phase was washed with brine $(20 \mathrm{~mL})$ and dried over anhydrous $\mathrm{MgSO}_{4}$. After removal of the solvent under reduced pressure, the crude was purified by column chromatography on silica gel with ethyl acetate - hexane $(1: 10, \mathrm{v}: \mathrm{v})$ as the eluent to give the pure alcohol 1a as a colorless oil. Yield: $1.82 \mathrm{~g}$ (79\%). IR (film): $v\left(\mathrm{~cm}^{-1}\right)$ 3392, 2925, 2854, 1630, 1465, 1131, 887. ${ }^{1} \mathrm{H}$ NMR $\left(300 \mathrm{MHz}, \mathrm{CDCl}_{3}\right) \delta$ 0.86-0.90 (3H, m), $1.27(13 \mathrm{H}, \mathrm{s}), 1.49(3 \mathrm{H}, \mathrm{s}), 1.70(1 \mathrm{H}, \mathrm{br})$, 2.46-2.59 (2H, m), 3.92-3.96 (1H, m), $5.54(1 \mathrm{H}, \mathrm{s}), 5.69(1 \mathrm{H}, \mathrm{s}) .{ }^{13} \mathrm{C} \mathrm{NMR}\left(75.4 \mathrm{MHz}, \mathrm{CDCl}_{3}\right)$ $\delta$ 14.1, 22.6, 25.6, 29.3, 29.5, 29.6, 31.9, 36.4, 49.4, 69.1, 119.4, 130.9. EIMS: $\mathrm{m} / z$ (rel intensity) $157\left(\mathrm{M}^{+}-119,4\right), 122$ (1), 111 (1), 97 (24), 83 (77), 69 (62), 55 (100), 41 (72). Anal. calcd for $\mathrm{C}_{13} \mathrm{H}_{25} \mathrm{BrO}: \mathrm{C}, 56.32 ; \mathrm{H}, 9.09$. Found: $\mathrm{C}, 56.48 ; \mathrm{H}, 8.87$.

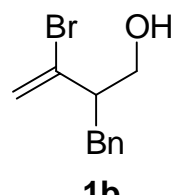

Substrate 1b was prepared by the reduction of ethyl 2-benzyl-3-bromobut-3-enoate with $\mathrm{LiAlH}_{4}{ }^{2}{ }^{2}$ The ether solution $(5 \mathrm{~mL})$ of the above ester $(1.44 \mathrm{~g}, 5.09 \mathrm{mmol})$ was added dropwise into the mixture of $\mathrm{LiAlH}_{4}(213 \mathrm{mg}, 5.60 \mathrm{mmol})$ and ether $(20 \mathrm{~mL})$ in a $50 \mathrm{~mL}$ three-necked round flask at room temperature. The addition brought the mixture to reflux. After the addition was complete, the resulting mixture was stirred at room temperature for another $5 \mathrm{~h}$. The reaction flask was then cooled with an ice bath and wet ether was added slowly into the 
mixture to destroy the excess $\mathrm{LiAlH}_{4}$. Dilute hydrochloric acid $(10 \mathrm{~mL})$ was then added and the two layers were seperated. The aqueous phase was extracted with ether $(3 \times 20 \mathrm{~mL})$. The combined organic phase was washed with brine $(10 \mathrm{~mL})$ and then dried over anhydrous $\mathrm{MgSO}_{4}$. After removal of the solvent under reduced pressure, the crude was purified by column chromatography on silica gel with ethyl acetate - hexane $(1: 5, \mathrm{v}: \mathrm{v})$ as the eluent to give the pure alcohol $\mathbf{1 b}$ as a colorless oil. Yield: $1.03 \mathrm{~g}(84 \%)$. IR (film): $v\left(\mathrm{~cm}^{-1}\right)$ 3371, 3027, 2934, 2880, 1623, 1496, 1454, 1050, 1031, 892, 746, 700. ${ }^{1} \mathrm{H}$ NMR $\left(300 \mathrm{MHz}, \mathrm{CDCl}_{3}\right) \delta 1.56(1 \mathrm{H}$, br), 2.70-2.78 (3H, m), 3.64-3.69 (2H, m), $5.52(1 \mathrm{H}, \mathrm{d}, J=1.8 \mathrm{~Hz}), 5.59(1 \mathrm{H}, \mathrm{d}, J=1.5 \mathrm{~Hz})$, 7.18-7.23 (3H, m), 7.26-7.31 (2H, m). $\left.{ }^{13} \mathrm{C} \mathrm{NMR} \mathrm{(75.4} \mathrm{MHz,} \mathrm{CDCl}_{3}\right) \delta 36.0,54.3,63.5,120.1$, 126.3, 128.3, 129.0, 135.3, 138.7. EIMS: $m / z$ (rel intensity) $240\left(\mathrm{M}^{+}, 0.02\right), 222(1), 143(35)$, 129 (9), 108 (7), 91 (100), 78 (4), 51 (7). Anal. calcd for $\mathrm{C}_{11} \mathrm{H}_{13} \mathrm{BrO}$ : C, 54.79; H, 5.43. Found: C, 54.79; H, 5.36.

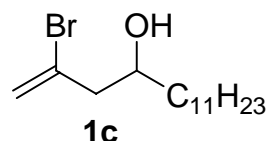

Compound 1c was prepared by the Barbier reaction of 2,3-dibromoprop-1-ene with dodecanal according to the procedure outlined for the synthesis of 1a. Yield: $60 \%$. Colorless oil. IR (film): $v\left(\mathrm{~cm}^{-1}\right) 3393,2926,2854,1627,1464,884 .{ }^{1} \mathrm{H}$ NMR (300 MHz, $\left.\mathrm{CDCl}_{3}\right) \delta$ 0.86-0.90 (3H, m), $1.26(17 \mathrm{H}, \mathrm{s}), 1.49(3 \mathrm{H}, \mathrm{s}), 1.71(1 \mathrm{H}, \mathrm{d}, J=3.3 \mathrm{~Hz}), 2.46-2.60(2 \mathrm{H}, \mathrm{m}), 3.92-3.96(1 \mathrm{H}, \mathrm{m})$, $5.54(1 \mathrm{H}, \mathrm{d}, J=1.5 \mathrm{~Hz}), 5.70(1 \mathrm{H}, \mathrm{s}) .{ }^{13} \mathrm{C} \mathrm{NMR}\left(75.4 \mathrm{MHz}, \mathrm{CDCl}_{3}\right) \delta 14.1,22.7,25.6,29.3$, 29.59, 29.63, 29.7, 31.9, 36.4, 49.4, 69.1, 119.5, 130.9. EIMS: $m / z$ (rel intensity) $225\left(\mathrm{M}^{+}-\mathrm{Br}\right.$, 1), 185 (6), 125 (5), 111 (33), 97 (52), 83 (64), 69 (100), 41 (53). Anal. calcd for $\mathrm{C}_{15} \mathrm{H}_{29} \mathrm{BrO}$ : C, 59.01; H, 9.57. Found: C, 59.40; H, 9.41.

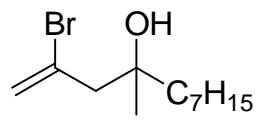

1d

Compound 1d was prepared by the Barbier reaction of 2,3-dibromoprop-1-ene with octanal (76\% yield) according to the procedure outlined in the preparation of $\mathbf{1 a}$ followed by the conventional oxidation with pyridinium chlorochromate ${ }^{3}(79 \%$ yield) and the subsequent Grignard reaction ${ }^{4}$ with methylmagnesium bromide ( $41 \%$ yield). Colorless oil. IR (film): v 
$\left(\mathrm{cm}^{-1}\right) 2956,2929,2855,1624,1465,1377,1154,890 .{ }^{1} \mathrm{H}$ NMR $\left(300 \mathrm{MHz}, \mathrm{CDCl}_{3}\right) \delta$ 0.86-0.90 (3H, m), 1.25-1.38 (13H, m), 1.50-1.55 (2H, m), $1.82(1 \mathrm{H}, \mathrm{s}), 2.67\left(2 \mathrm{H}, \mathrm{dd}, J_{1}=14.4\right.$ $\left.\mathrm{Hz}, J_{2}=19.5 \mathrm{~Hz}\right), 5.62(1 \mathrm{H}, \mathrm{d}, J=1.5 \mathrm{~Hz}), 5.66(1 \mathrm{H}, \mathrm{s}) .{ }^{13} \mathrm{C} \mathrm{NMR}\left(75.4 \mathrm{MHz}, \mathrm{CDCl}_{3}\right) \delta 14.1$, 22.6, 23.9, 26.7, 29.3, 30.0, 31.8, 42.1, 52.2, 72.5, 121.1, 128.6. EIMS: $m / z$ (rel intensity) 245 (M+17, 1), 207 (2), 175 (2), 143 (16), 127 (23), 83 (34), 57 (83), 43 (100). HRMS calcd for $\mathrm{C}_{12} \mathrm{H}_{21} \mathrm{Br}\left(\mathrm{M}^{+}-\mathrm{H}_{2} \mathrm{O}\right): 244.0827$. Found: 244.0829.

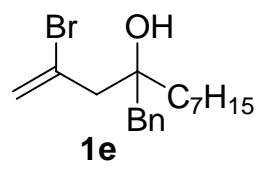

Compound 1e was prepared by the Barbier reaction of 2,3-dibromoprop-1-ene with octanal (76\% yield) according to the procedure outlined in the preparation of $\mathbf{1 a}$ followed by the conventional oxidation with pyridinium chlorochromate ${ }^{3}(79 \%$ yield) and the subsequent Grignard reaction $^{4}$ with benzylmagnesium bromide (32\% yield). Colorless oil. IR (film): $v$ $\left(\mathrm{cm}^{-1}\right)$ 3576, 3028, 2927, 2855, 1623, 1495, 1454, 1377, 1142, 891, 702. ${ }^{1} \mathrm{H}$ NMR (300 MHz, $\left.\mathrm{CDCl}_{3}\right) \delta$ 0.86-0.88 (3H, m), $1.27(8 \mathrm{H}, \mathrm{s}), 1.40-1.56(4 \mathrm{H}, \mathrm{m}), 1.75(1 \mathrm{H}, \mathrm{t}, J=2.1 \mathrm{~Hz})$, 2.60-2.74 (2H, m), $2.83(2 \mathrm{H}, \mathrm{d}, J=3.3 \mathrm{~Hz}), 5.63-5.64(1 \mathrm{H}, \mathrm{m}), 5.69(1 \mathrm{H}, \mathrm{s}), 7.22-7.30(5 \mathrm{H}, \mathrm{m})$. ${ }^{13} \mathrm{C}$ NMR $\left(75.4 \mathrm{MHz}, \mathrm{CDCl}_{3}\right) \delta 14.1,22.6,23.9,29.2,29.9,31.7,38.6,45.3,49.6,74.2,121.4$, 126.5, 128.1, 128.3, 130.6, 136.8. EIMS: $m / z$ (rel intensity) $323\left(\mathrm{M}^{+}-15,0.1\right), 241$ (4), 219 (19), 143 (2), 127 (94), 91 (59), 57 (100), 43 (36). HRMS calcd for $\mathrm{C}_{18} \mathrm{H}_{26} \mathrm{Br}\left(\mathrm{M}^{+}-\mathrm{OH}\right): 321.1218$. Found: 321.1212.

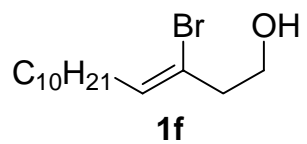

Compound 1f was prepared by the reduction of (Z)-ethyl 3-bromotetradec-3-enoate with $\mathrm{LiAlH}_{4}$ according to the procedure outlined for the preparation of $\mathbf{1 b}$. Yield: 80\%. Colorless oil. IR (film): v (cm $\left.{ }^{-1}\right)$ 3348, 2924, 2854, 1658, 1465, 1378, 1046. ${ }^{1} \mathrm{H}$ NMR $\left(300 \mathrm{MHz}, \mathrm{CDCl}_{3}\right) \delta$ 0.85-0.89 (3H, m), $1.26(16 \mathrm{H}, \mathrm{s}), 1.39(1 \mathrm{H}, \mathrm{br}), 2.13-2.18(2 \mathrm{H}, \mathrm{m}), 2.65-2.67(2 \mathrm{H}, \mathrm{m})$, 3.77-3.79 (2H, m), 5.75-5.79 (1H, m). ${ }^{13} \mathrm{C}$ NMR (75.4 MHz, $\left.\mathrm{CDCl}_{3}\right) \delta 14.1,22.7,28.4,29.2$, 29.3, 29.4, 29.6, 31.4, 31.9, 44.6, 60.3, 123.6, 132.0. EIMS: $\mathrm{m} / z$ (rel intensity) $290\left(\mathbf{M}^{+}, 0.3\right)$, 254 (2), 211 (7), 146 (18), 109 (35), 81 (61), 55 (83), 43 (100). Anal. calcd for $\mathrm{C}_{14} \mathrm{H}_{27} \mathrm{BrO}$ : C, 
57.73; H, 9.34. Found: C, 57.73; H, 9.18.

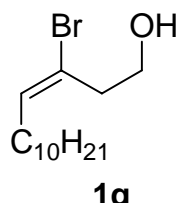

Compound 1g was prepared by the reduction of (E)-ethyl 3-bromotetradec-3-enoate with $\mathrm{LiAlH}_{4}$ according to the procedure outlined for the preparation of $\mathbf{1 b}$. Yield: 80\%. Colorless oil. IR (film): v (cm $\left.{ }^{-1}\right) 3341,2955,2924,2854,1642,1465,1047 .{ }^{1} \mathrm{H}$ NMR $\left(300 \mathrm{MHz}, \mathrm{CDCl}_{3}\right) \delta$ 0.82-0.88 (3H, m), $1.25(16 \mathrm{H}, \mathrm{s}), 1.57(1 \mathrm{H}, \mathrm{br}), 2.00-2.10(2 \mathrm{H}, \mathrm{m}), 2.65-2.72(2 \mathrm{H}, \mathrm{m})$, 3.76-3.83 (2H, m), 5.98-6.06 (1H, m). ${ }^{13} \mathrm{C} \mathrm{NMR}\left(75.4 \mathrm{MHz}, \mathrm{CDCl}_{3}\right) \delta 14.2,22.7,29.1,29.2$, 29.3, 29.4, 29.5, 29.6, 29.8, 31.9, 38.7, 60.5, 120.9, 135.7. EIMS: $m / z$ (rel intensity) $290\left(\mathrm{M}^{+}\right.$, 0.2), 211 (7), 146 (26), 109 (34), 95 (58), 81 (56), 55 (76), 43 (100). Anal. calcd for $\mathrm{C}_{14} \mathrm{H}_{27} \mathrm{BrO}$ : C, 57.73; H, 9.34. Found: C, 57.82; H, 9.26.

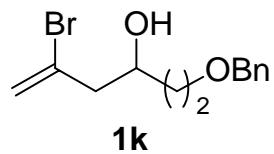

Compound 1k was prepared by the Barbier reaction of 2,3-dibromoprop-1-ene with 3-(benzyloxy)propanal according to the procedure outlined for the synthesis of 1a. Yield: 74\%. Colorless oil. IR (film): v ( $\left.\mathrm{cm}^{-1}\right)$ 3443, 3063, 3030, 2916, 2862, 1735, 1630, 1496, 1454, 1203, 1098, 1027, 891, 738, 698. ${ }^{1} \mathrm{H}$ NMR (300 MHz, $\left.\mathrm{CDCl}_{3}\right) \delta 1.77-1.83(2 \mathrm{H}, \mathrm{m}), 2.52\left(1 \mathrm{H}, \mathrm{dd}, J_{1}\right.$ $\left.=4.8 \mathrm{~Hz}, J_{2}=14.4 \mathrm{~Hz}\right), 2.63\left(1 \mathrm{H}, \mathrm{dd}, J_{1}=4.8 \mathrm{~Hz}, J_{2}=14.4 \mathrm{~Hz}\right), 3.03(1 \mathrm{H}, \mathrm{d}, J=2.4 \mathrm{~Hz})$, 3.64-3.76 (2H, m), 4.15-4.19 (1H, m), $4.53(2 \mathrm{H}, \mathrm{s}), 5.52(1 \mathrm{H}, \mathrm{s}), 5.68(1 \mathrm{H}, \mathrm{s}), 7.27-7.35(5 \mathrm{H}$, m). ${ }^{13} \mathrm{C}$ NMR $\left(75.4 \mathrm{MHz}, \mathrm{CDCl}_{3}\right) \delta 35.5,49.1,68.7,73.4,119.3,127.7,127.8,128.5,130.5$, 137.8. EIMS: $m / z$ (rel intensity) 187 (M-97+, 1), 165 (1), 146 (2), 120 (3), 107 (14), 91 (100), 79 (8), 41 (9). Anal. calcd for $\mathrm{C}_{13} \mathrm{H}_{17} \mathrm{BrO}_{2}$ : C, 54.75; H, 6.01. Found: C, 55.09; H, 6.07.

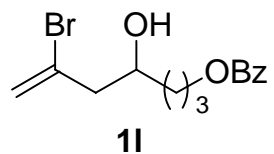

Compound 11 was prepared by the Barbier reaction of 2,3-dibromoprop-1-ene with 4-oxobutyl 
benzoate according to the procedure outlined for the synthesis of 1a. Yield: 40\%. Colorless oil. IR (film): $v\left(\mathrm{~cm}^{-1}\right)$ 3480, 2952, 1716, 1631, 1452, 1277, 1117, 1070, 1026, 891, 712. ${ }^{1} \mathrm{H}$ NMR $\left(300 \mathrm{MHz}, \mathrm{CDCl}_{3}\right) \delta$ 1.67-1.73 (2H, m), 1.84-2.03 (3H, m), 2.56-2.58 (2H, m), $4.03(1 \mathrm{H}, \mathrm{br})$, $4.37(2 \mathrm{H}, \mathrm{t}, J=6.0 \mathrm{~Hz}), 5.55(1 \mathrm{H}, \mathrm{s}), 5.71(1 \mathrm{H}, \mathrm{s}), 7.44(2 \mathrm{H}, \mathrm{t}, J=7.2 \mathrm{~Hz}), 7.56(1 \mathrm{H}, \mathrm{t}, J=7.2$ $\mathrm{Hz}), 8.05(2 \mathrm{H}, \mathrm{d}, J=7.2 \mathrm{~Hz}) .{ }^{13} \mathrm{C} \mathrm{NMR}\left(75.4 \mathrm{MHz}, \mathrm{CDCl}_{3}\right) \delta 25.0,32.7,49.4,64.8,68.5$, 119.8, 128.3, 129.5, 130.1, 130.4, 132.9, 166.6. EIMS: $m / z$ (rel intensity) $193\left(\mathrm{M}^{+}-119,8\right), 174$ (1), 123 (11), 105 (100), 77 (33), 71 (83), 51 (7), 41 (10). Anal. calcd for $\mathrm{C}_{14} \mathrm{H}_{17} \mathrm{BrO}_{3}$ : C, 53.69 ; H, 5.47. Found: C, 53.67; H, 5.56.

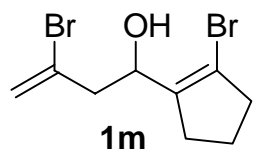

Compound 1m was prepared by the Barbier reaction of 2,3-dibromoprop-1-ene with 2-bromocyclopent-1-enecarbaldehyde according to the procedure outlined for the synthesis of 1a. Yield: $86 \%$. Pale yellow solid. Mp: $42-44{ }^{\circ} \mathrm{C}$. IR $\left(\mathrm{CH}_{2} \mathrm{Cl}_{2}\right): v\left(\mathrm{~cm}^{-1}\right) 3373,2925,2847$, $1652,1631,1471,1411,1200,1124,1052,891,859,587 .{ }^{1} \mathrm{H}$ NMR $\left(300 \mathrm{MHz}, \mathrm{CDCl}_{3}\right) \delta$ 1.91-2.01 (3H, m), 2.27-2.37 (1H, m), 2.47-2.76 (5H, m), 4.89-4.93 (1H, m), $5.56(1 \mathrm{H}, \mathrm{s}), 5.72$ $(1 \mathrm{H}, \mathrm{s}) .{ }^{13} \mathrm{C} \mathrm{NMR}\left(75.4 \mathrm{MHz}, \mathrm{CDCl}_{3}\right) \delta 21.6,29.9,40.2,46.6,67.4,118.2,119.7,129.6,140.8$. EIMS: $m / z$ (rel intensity) $295\left(\mathrm{M}^{+}+1,1\right), 251$ (1), 175 (59), 117 (3), 96 (38), 79 (8), 67 (100), 41 (33). Anal. calcd for $\mathrm{C}_{9} \mathrm{H}_{12} \mathrm{Br}_{2} \mathrm{O}$ : C, 36.52; H, 4.09. Found: C, 36.39; H, 4.12.

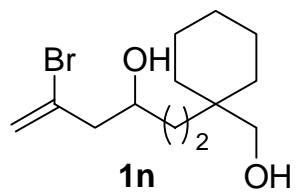

Compound 1n was prepared by the Barbier reaction of 2,3-dibromoprop-1-ene with ethyl 1-(3-oxopropyl)cyclohexanecarboxylate according to the procedure outlined for the synthesis of $1 \mathbf{a}$ followed by the reduction with $\mathrm{LiAlH}_{4}$ according to the procedure outlined for the preparation of 1b. Overall yield: $77 \%$. White solid. Mp: $84{ }^{\circ} \mathrm{C}$. IR $\left(\mathrm{CH}_{2} \mathrm{Cl}_{2}\right): v\left(\mathrm{~cm}^{-1}\right) 3364$, 3296, 2929, 2844, 1631, 1447, 1205, 1120, 1081, 1036, 982, 888. ${ }^{1} \mathrm{H}$ NMR (300 MHz, $\mathrm{CDCl}_{3}$ ) $\delta$ 1.30-1.33 (4H, m), 1.36-1.55 (10H, m), $2.18(2 \mathrm{H}, \mathrm{s}), 2.54-2.57(2 \mathrm{H}, \mathrm{m}), 3.37-3.45(2 \mathrm{H}, \mathrm{m})$, 3.91-3.93 $(1 \mathrm{H}, \mathrm{m}), 5.54(1 \mathrm{H}, \mathrm{d}, J=1.5 \mathrm{~Hz}), 5.70(1 \mathrm{H}, \mathrm{s}) .{ }^{13} \mathrm{C} \mathrm{NMR}\left(75.4 \mathrm{MHz}, \mathrm{CDCl}_{3}\right) \delta 21.5$, 
26.4, 29.4, 29.8, 32.5, 32.9, 36.9, 49.4, 67.9, 69.8, 119.6, 130.8. EIMS: $m / z$ (rel intensity) 262 (M-28+ , 1), 171 (32), 153 (37), 135 (100), 121 (31), 95 (45), 79 (28), 41 (25). Anal. calcd for $\mathrm{C}_{13} \mathrm{H}_{23} \mathrm{BrO}_{2}$ : C, 53.61; H, 7.96. Found: C, 53.45; H, 8.19.

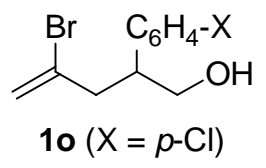

Compound 10 was prepared by the $\mathrm{LiAlH}_{4}$ reduction of ethyl 4-bromo-2-(4-chlorophenyl)pent-4-enoate according to the procedure outlined for the preparation of 1b. Yield: $74 \%$. Colorless oil. IR (film): $v\left(\mathrm{~cm}^{-1}\right) 3374,2930,2876,1896,1630,1492,1092,1014,891,825 .{ }^{1} \mathrm{H}$ NMR (300 MHz, $\left.\mathrm{CDCl}_{3}\right) \delta 1.57(1 \mathrm{H}, \mathrm{br}), 2.61-2.69(1 \mathrm{H}, \mathrm{m}), 2.83-2.90(1 \mathrm{H}, \mathrm{m}), 3.19-3.24(1 \mathrm{H}$, m), 3.76-3.78 (2H, m), $5.35(1 \mathrm{H}, \mathrm{d}, J=1.8 \mathrm{~Hz}), 5.44(1 \mathrm{H}, \mathrm{d}, J=1.2 \mathrm{~Hz}), 7.17\left(2 \mathrm{H}, \mathrm{dd}, J_{1}=1.8\right.$ $\left.\mathrm{Hz}, J_{2}=8.1 \mathrm{~Hz}\right), 7.31\left(2 \mathrm{H}, \mathrm{dd}, J_{1}=1.8 \mathrm{~Hz}, J_{2}=8.1 \mathrm{~Hz}\right) .{ }^{13} \mathrm{C} \mathrm{NMR}\left(75.4 \mathrm{MHz}, \mathrm{CDCl}_{3}\right) \delta 43.7$, 45.7, 65.8, 118.9, 128.8, 129.4, 131.6, 132.7, 139.2. EIMS: $m / z$ (rel intensity) $257\left(\mathrm{M}^{+}-17,2\right)$, 245 (9), 195 (4), 177 (32), 155 (100), 128 (67), 91 (64), 43 (13). Anal. calcd for $\mathrm{C}_{11} \mathrm{H}_{12} \mathrm{BrClO}$ : C, 47.94; H, 4.39. Found: C, 48.31; H, 4.63.

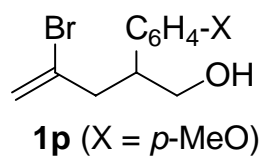

Compound 1p was prepared by the $\mathrm{LiAlH}_{4}$ reduction of ethyl 4-bromo-2-(4-methoxyphenyl)pent-4-enoate according to the procedure outlined for the preparation of $\mathbf{1 b}$. Yield: $80 \%$. Colorless oil. IR (film): v (cm $\left.{ }^{-1}\right)$ 3397, 2933, 2835, 1630, 1611, 1513, 1249, 1179, 1107, 1034, 890, 829. ${ }^{1} \mathrm{H}$ NMR (300 MHz, $\left.\mathrm{CDCl}_{3}\right) \delta 1.59(1 \mathrm{H}, \mathrm{br}), 2.65\left(1 \mathrm{H}, \mathrm{dd}, J_{1}=8.4 \mathrm{~Hz}, J_{2}=14.4 \mathrm{~Hz}\right)$, $2.84\left(1 \mathrm{H}, \mathrm{dd}, J_{1}=6.3 \mathrm{~Hz}, J_{2}=14.1 \mathrm{~Hz}\right), 3.16-3.21(1 \mathrm{H}, \mathrm{m}), 3.74(2 \mathrm{H}, \mathrm{d}, J=6.3 \mathrm{~Hz}), 3.78(3 \mathrm{H}$, s), $5.34(1 \mathrm{H}, \mathrm{d}, J=1.5 \mathrm{~Hz}), 5.44(1 \mathrm{H}, \mathrm{d}, J=1.2 \mathrm{~Hz}), 6.87\left(2 \mathrm{H}, \mathrm{dd}, J_{1}=1.8 \mathrm{~Hz}, J_{2}=6.9 \mathrm{~Hz}\right)$, 7.12-7.16 (2H, m). ${ }^{13} \mathrm{C}$ NMR (75.4 MHz, $\left.\mathrm{CDCl}_{3}\right) \delta 43.9,45.4,55.1,66.1,113.9,114.0,118.6$, 128.9, 132.0, 132.4, 158.4. EIMS: $m / z$ (rel intensity) $270\left(\mathrm{M}^{+}, 4\right), 239$ (6), 192 (1), 151 (100), 121 (26), 91 (45), 77 (18), 51 (7). HRMS calcd for $\mathrm{C}_{12} \mathrm{H}_{15} \mathrm{BrO}_{2}$ : 270.0255. Found: 270.0253. 
<smiles>C=C(Br)CCC(C)(O)Br</smiles>

Compound 1q was prepared by the Grignard reaction of 5-bromohex-5-en-2-one with benzylmagnesium bromide according to the conventional procedure. ${ }^{4}$ Yield: $54 \%$. Colorless oil. IR (film): $v\left(\mathrm{~cm}^{-1}\right)$ 3565, 3447, 3028, 2970, 2924, 1630, 1494, 1453, 1088, 908, 885, 732, 702. ${ }^{1} \mathrm{H}$ NMR (300 MHz, $\left.\mathrm{CDCl}_{3}\right) \delta 1.17(3 \mathrm{H}, \mathrm{s}), 1.34(1 \mathrm{H}, \mathrm{s}), 1.76(2 \mathrm{H}, \mathrm{t}, J=8.4 \mathrm{~Hz})$, 2.58-2.62 (2H, m), $2.78\left(2 \mathrm{H}, \mathrm{dd}, J_{1}=13.2 \mathrm{~Hz}, J_{2}=21.9 \mathrm{~Hz}\right), 5.39(1 \mathrm{H}, \mathrm{s}), 5.59(1 \mathrm{H}, \mathrm{s})$, 7.21-7.35 (5H, m). ${ }^{13} \mathrm{C}$ NMR (75.4 MHz, $\left.\mathrm{CDCl}_{3}\right) \delta 26.3,36.2,40.1,48.2,71.8,116.3,126.6$, 128.2, 130.5, 134.7, 136.9. EIMS: $m / z$ (rel intensity) $253\left(\mathrm{M}^{+}-\mathrm{CH}_{3}, 0.2\right), 177$ (5), 135 (11), 117 (5), 97 (66), 91 (52), 77 (5), 43 (100). HRMS calcd for $\mathrm{C}_{12} \mathrm{H}_{14} \mathrm{BrO}\left(\mathrm{M}^{+}-\mathrm{CH}_{3}\right)$ : 253.0228. Found: 253.0240.

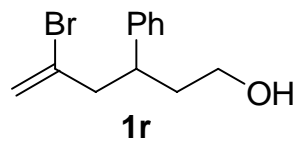

Compound 1r was prepared by the reduction of 5-bromo-3-phenylhex-5-enal with $\mathrm{LiAlH}_{4}$ according to the procedure outlined for the preparation of $\mathbf{1 b}$. Yield: $75 \%$. Colorless oil. IR (film): $v\left(\mathrm{~cm}^{-1}\right) 3348,3028,2933,1629,1494,1453,1430,1204,1125,1045,888,701 .{ }^{1} \mathrm{H}$ NMR $\left(300 \mathrm{MHz}, \mathrm{CDCl}_{3}\right) \delta 1.35(1 \mathrm{H}, \mathrm{s}), 1.71-1.87(1 \mathrm{H}, \mathrm{m}), 1.93-2.04(1 \mathrm{H}, \mathrm{m}), 2.70(2 \mathrm{H}, \mathrm{d}, J=$ $7.2 \mathrm{~Hz}), 3.11-3.21(1 \mathrm{H}, \mathrm{m}), 3.45-3.52(2 \mathrm{H}, \mathrm{m}), 5.31(1 \mathrm{H}, \mathrm{d}, J=1.8 \mathrm{~Hz}), 5.40(1 \mathrm{H}, \mathrm{s}), 7.18-7.26$ $(3 \mathrm{H}, \mathrm{m}), 7.28-7.33(2 \mathrm{H}, \mathrm{m}) .{ }^{13} \mathrm{C}$ NMR $\left(75.4 \mathrm{MHz}, \mathrm{CDCl}_{3}\right) \delta 37.7,40.3,48.5,60.5,118.4$, $126.5,127.3,127.5,128.4,132.2,142.9 . \mathrm{m} / z$ (rel intensity) $236\left(\mathrm{M}^{+}-\mathrm{H}_{2} \mathrm{O}, 1\right), 175(1), 157$ (7), 135 (37), 117 (17), 105 (100), 91 (55), 51 (8). Anal. calcd for $\mathrm{C}_{12} \mathrm{H}_{15} \mathrm{BrO}$ : C, 56.49; H, 5.93. Found: C, 56.68; H, 5.96.<smiles>OCC1(CC2=C(Br)CCCC2)CCCCC1</smiles>

Compound 1s was prepared by the reduction of methyl 1-((2-bromocyclohex-1-enyl)methyl)cyclohexanecarboxylate with $\mathrm{LiAlH}_{4}$ according to the procedure outlined for the preparation of 1b. Yield: 85\%. Colorless oil. IR (film): $v\left(\mathrm{~cm}^{-1}\right)$ 3419, 2929, 2858, 1448, 1330, 1040, 972. 
${ }^{1} \mathrm{H}$ NMR $\left(300 \mathrm{MHz}, \mathrm{CDCl}_{3}\right) \delta$ 1.29-1.41 (10H, m), 1.59-1.65 (5H, m), $2.13(2 \mathrm{H}, \mathrm{s}), 2.22(2 \mathrm{H}$, s), $2.48(2 \mathrm{H}, \mathrm{s}), 3.45(2 \mathrm{H}, \mathrm{s}) .{ }^{13} \mathrm{C}$ NMR $\left(75.4 \mathrm{MHz} \mathrm{CDCl}_{3}\right) \delta 21.7,22.8,24.9,26.1,33.5,33.8$, 37.3, 39.7, 44.6, 67.2, 122.2, 134.8. EIMS: $m / z$ (rel intensity) $287\left(\mathbf{M}^{+}+1,0.02\right), 207$ (6), 174 (7), 111 (9), 95 (100), 77 (17), 55 (15), 41 (30). Anal. calcd for $\mathrm{C}_{14} \mathrm{H}_{23} \mathrm{BrO}$ : C, 58.54; H, 8.07. Found: C, 58.51; H, 8.12.

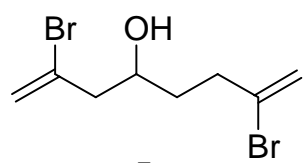

$5 a$

Compound 5a was prepared by the Barbier reaction of 2,3-dibromoprop-1-ene with 4-bromopent-4-enal according to the procedure outlined for the synthesis of 1a. Yield: $40 \%$. Colorless oil. IR (film): $v\left(\mathrm{~cm}^{-1}\right) 3393,2920,1630,1430,1188,1115,1064,889 .{ }^{1} \mathrm{H}$ NMR (300 $\left.\mathrm{MHz}, \mathrm{CDCl}_{3}\right) \delta 1.66-1.83(3 \mathrm{H}, \mathrm{m}), 2.56-2.65(4 \mathrm{H}, \mathrm{m}), 3.96-3.99(1 \mathrm{H}, \mathrm{m}), 5.43(1 \mathrm{H}, \mathrm{d}, J=1.5$ $\mathrm{Hz}), 5.56(1 \mathrm{H}, \mathrm{d}, J=1.5 \mathrm{~Hz}), 5.63(1 \mathrm{H}, \mathrm{d}, J=1.5 \mathrm{~Hz}), 5.71(1 \mathrm{H}, \mathrm{s}) .{ }^{13} \mathrm{C}$ NMR $(75.4 \mathrm{MHz}$, $\left.\mathrm{CDCl}_{3}\right) \delta 34.6,37.7,49.5,67.9,117.0,119.9,130.2,133.9$. EIMS: $m / z$ (rel intensity) $282\left(\mathrm{M}^{+}\right.$, 1), 265 (2), 201 (12), 161 (11), 133 (19), 93 (37), 79 (36), 41 (100). Anal. calcd for $\mathrm{C}_{8} \mathrm{H}_{12} \mathrm{Br}_{2} \mathrm{O}$ : C, 33.83; H, 4.26. Found: C, 34.04; H, 4.26.

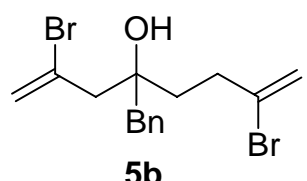

Compound $\mathbf{5 b}$ was prepared by the Dess-Martin oxidation ${ }^{5}$ of $\mathbf{5 a}$ (99\% yield) followed by the Grignard reaction ${ }^{4}$ with benzylmagnesium bromide ( $51 \%$ yield) according to the conventional procedures. Colorless oil. IR (film): $v\left(\mathrm{~cm}^{-1}\right)$ 3565, 3028, 2924, 1627, 1495, 1453, 1136, 1083, 890, 742, 703. ${ }^{1} \mathrm{H}$ NMR (300 MHz, $\left.\mathrm{CDCl}_{3}\right) \delta$ 1.76-1.87 (3H, m), 2.60-2.63 (2H, m), 2.69 (2H, $\mathrm{d}, J=2.1 \mathrm{~Hz}), 2.86(2 \mathrm{H}, \mathrm{s}), 5.39(1 \mathrm{H}, \mathrm{s}), 5.58(1 \mathrm{H}, \mathrm{s}), 5.67(1 \mathrm{H}, \mathrm{d}, J=1.8 \mathrm{~Hz}), 5.72(1 \mathrm{H}, \mathrm{s})$, 7.26-7.35 (5H, m). ${ }^{13} \mathrm{C}$ NMR (75.4 MHz, $\left.\mathrm{CDCl}_{3}\right) \delta 36.0,37.2,45.5,49.8,73.5,116.6,122.0$, 126.8, 127.7, 128.4, 130.7, 134.1, 136.2. EIMS: $m / z$ (rel intensity) $293\left(\mathbf{M}^{+}-\mathrm{Br}, 0.2\right), 255$ (3), 201 (7), 161 (34), 133 (36), 91 (100), 65 (17), 43 (18). HRMS calcd for $\mathrm{C}_{15} \mathrm{H}_{18} \mathrm{BrO}\left(\mathrm{M}^{+}\right.$-Br): 293.0541. Found: 293.0535. 


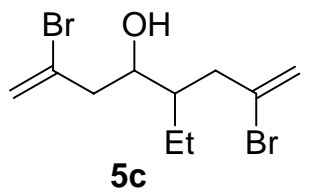

Compound $\mathbf{5 c}$ was prepared by the Barbier reaction of 2,3-dibromoprop-1-ene with 4-bromo-2-ethylpent-4-enal according to the procedure outlined for the synthesis of 1a. Yield: $50 \%$. Two stereoisomers in a 2:1 ratio determined by ${ }^{1} \mathrm{H}$ NMR $(300 \mathrm{MHz})$. Colorless oil. IR (film): $v\left(\mathrm{~cm}^{-1}\right)$ 3465, 2962, 2933, 2876, 1629, 1462, 1431, 1144, 1028, 889. ${ }^{1} \mathrm{H}$ NMR (300 $\left.\mathrm{MHz}, \mathrm{CDCl}_{3}\right) \delta 0.96(3 \mathrm{H}, \mathrm{t}, J=7.2 \mathrm{~Hz}), 1.33-1.54(2 \mathrm{H}, \mathrm{m}), 1.59-1.68(1 \mathrm{H}, \mathrm{m}), 1.78-1.84(1 \mathrm{H}$, m), 2.37-2.45 (1H, m), 2.53-2.64 (3H, m), $4.06(1 \mathrm{H}, \mathrm{d}, J=3.3 \mathrm{~Hz}), 5.46,5.49(1 \mathrm{H}, \mathrm{s}), 5.56(1 \mathrm{H}$, d, $J=0.9 \mathrm{~Hz}), 5.63,5.65(1 \mathrm{H}, \mathrm{s}), 5.73(1 \mathrm{H}, \mathrm{s}) .{ }^{13} \mathrm{C} \mathrm{NMR}\left(75.4 \mathrm{MHz}, \mathrm{CDCl}_{3}\right) \delta 11.2 / 11.7$, 20.9/22.2, 41.1/42.1, 42.3/42.4, 46.3/46.5, 69.1/69.2, 118.3/118.4, 119.6/119.8, 131.0/131.1, 133.6/133.9. EIMS: $m / z$ (rel intensity) $310\left(\mathrm{M}^{+}, 1\right), 283$ (4), 222 (32), 147 (66), 111 (46), 91 (52), 77 (39), 43 (100). Anal. calcd for $\mathrm{C}_{10} \mathrm{H}_{16} \mathrm{Br}_{2} \mathrm{O}$ : C, 38.49; H, 5.17. Found: C, 38.62; H, 5.06 .<smiles>C=C(Br)CC(O)C(C)C/C(Br)=C/C</smiles>

Compound 5d was prepared by the Barbier reaction of 2,3-dibromoprop-1-ene with (Z)-4-bromo-2-methylhex-4-enal according to the procedure outlined for the synthesis of $\mathbf{1 a}$. Yield: 52\%. Two stereoisomers in a 3:2 ratio determined by ${ }^{1} \mathrm{H}$ NMR $(300 \mathrm{MHz})$. Colorless oil. IR (film): $v\left(\mathrm{~cm}^{-1}\right)$ 3446, 2965, 2915, 1660, 1630, 1458, 1430, 1379, 1291, 1136, 989, 891. ${ }^{1} \mathrm{H}$ NMR $\left(300 \mathrm{MHz}, \mathrm{CDCl}_{3}\right) \delta$ 0.87-0.93 $(3 \mathrm{H}, \mathrm{m}), 1.64(1 \mathrm{H}, \mathrm{br}), 1.73-1.81(3 \mathrm{H}, \mathrm{m}), 2.00-2.05(1 \mathrm{H}$, m), 2.21-2.39 $(1 \mathrm{H}, \mathrm{m}), 2.50-2.64(3 \mathrm{H}, \mathrm{m}), 3.83-3.94(1 \mathrm{H}, \mathrm{m})$, 5.55-5.56 $(1 \mathrm{H}, \mathrm{m})$, 5.71-5.80 $(2 \mathrm{H}, \mathrm{m}) .{ }^{13} \mathrm{C}$ NMR $\left(75.4 \mathrm{MHz}, \mathrm{CDCl}_{3}\right) \delta 12.3,14.9 / 16.9,35.7 / 36.3,43.9 / 45.5,46.1 / 47.0$, 70.5/71.9, 119.6/119.9, 124.90/125.94, 127.8, 131.0. EIMS: $m / z$ (rel intensity) $231\left(\mathrm{M}^{+}-\mathrm{Br}, 1\right)$, 213 (1), 173 (4), 133 (12), 111 (100), 83 (20), 53 (55), 41 (92). HRMS calcd for $\mathrm{C}_{10} \mathrm{H}_{16} \mathrm{BrO}$ ( $\left.\mathrm{M}^{+}-\mathrm{Br}\right):$ 231.0385. Found: 231.0383.

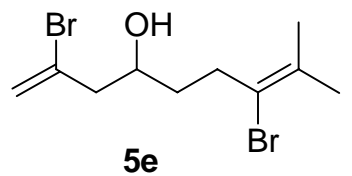


Compound 5e was prepared by the Barbier reaction of 2,3-dibromoprop-1-ene with 4-bromo-5-methylhex-4-enal according to the procedure outlined for the synthesis of 1a. Yield: 43\%. Colorless oil. IR (film): v ( $\left.\mathrm{cm}^{-1}\right)$ 3408, 2918, 2859, 1630, 1444, 1221, 1176, 1121, 1071, 999, 891. ${ }^{1} \mathrm{H}$ NMR (300 MHz, $\left.\mathrm{CDCl}_{3}\right) \delta 1.61-1.77(3 \mathrm{H}, \mathrm{m}), 1.80(3 \mathrm{H}, \mathrm{s}), 1.87(3 \mathrm{H}, \mathrm{s})$, 2.55-2.57 (2H, m), 2.64-2.69 (2H, m), 3.91-3.96 (1H, m), $5.55(1 \mathrm{H}, \mathrm{d}, J=1.2 \mathrm{~Hz}), 5.71(1 \mathrm{H}, \mathrm{s})$. ${ }^{13} \mathrm{C}$ NMR $\left(75.4 \mathrm{MHz}, \mathrm{CDCl}_{3}\right) \delta 20.4,25.4,33.9,34.9,49.5,68.2,119.8,121.0,130.4,130.9$. EIMS: $m / z$ (rel intensity) $231\left(\mathrm{M}^{+}-\mathrm{Br}, 2\right), 173$ (6), 147 (12), 111 (100), 93 (31), 79 (11), 67 (64), 41 (68). Anal. calcd for $\mathrm{C}_{10} \mathrm{H}_{16} \mathrm{Br}_{2} \mathrm{O}$ : C, 38.49; H, 5.17. Found: C, 38.56; H, 5.21.

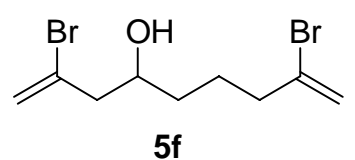

Compound 5f was prepared by the Barbier reaction of 2,3-dibromoprop-1-ene with 5-bromohex-5-enal according to the procedure outlined for the synthesis of 1a. Yield: $70 \%$. Colorless oil. IR (film): $v\left(\mathrm{~cm}^{-1}\right)$ 3392, 2941, 1630, 1430, 1134, 1081, 888. ${ }^{1} \mathrm{H}$ NMR (300 MHz, $\left.\mathrm{CDCl}_{3}\right) \delta$ 1.47-1.55 (2H, m), 1.59-1.85 (3H, m), 2.44-2.60 (4H, m), 3.94-3.97 (1H, m), 5.41 $(1 \mathrm{H}, \mathrm{d}, J=1.5 \mathrm{~Hz}), 5.55(1 \mathrm{H}, \mathrm{d}, J=2.4 \mathrm{~Hz}), 5.58-5.60(1 \mathrm{H}, \mathrm{m}), 5.71(1 \mathrm{H}, \mathrm{s}) .{ }^{13} \mathrm{C} \mathrm{NMR}(75.4$ $\left.\mathrm{MHz}, \mathrm{CDCl}_{3}\right) \delta 23.8,34.8,41.1,49.4,68.6,116.8,119.8,130.5,134.1$. EIMS: $\mathrm{m} / z$ (rel intensity) 217 (M+-Br, 1), 177 (2), 133 (22), 119 (7), 97 (40), 79 (63), 69 (49), 41 (100). HRMS calcd for $\mathrm{C}_{9} \mathrm{H}_{14} \mathrm{BrO}\left(\mathrm{M}^{+}-\mathrm{Br}\right)$ : 217.0228. Found: 217.0225.

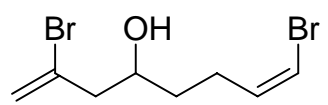

$5 \mathrm{~g}$

Compound 5g was prepared by the Barbier reaction of 2,3-dibromoprop-1-ene with (Z)-5-bromopent-4-enal according to the procedure outlined for the synthesis of 1a. Yield: 37\%. Colorless oil. IR (film): $v\left(\mathrm{~cm}^{-1}\right)$ 3401, 2933, 1629, 1434, 1306, 1287, 1120, 1075, 892. ${ }^{1} \mathrm{H}$ NMR $\left(300 \mathrm{MHz}, \mathrm{CDCl}_{3}\right) \delta$ 1.59-1.67 (2H, m), $1.81(1 \mathrm{H}, \mathrm{br}), 2.33-2.40(2 \mathrm{H}, \mathrm{m}), 2.53-2.61$ $(2 \mathrm{H}, \mathrm{m}), 3.93-3.99(1 \mathrm{H}, \mathrm{m}), 5.55(1 \mathrm{H}, \mathrm{s}), 5.71(1 \mathrm{H}, \mathrm{s}), 6.11-6.22(2 \mathrm{H}, \mathrm{m}) .{ }^{13} \mathrm{C} \mathrm{NMR}(75.4 \mathrm{MHz}$, $\left.\mathrm{CDCl}_{3}\right) \delta 26.0,34.4,49.2,68.3,108.4,119.8,130.3,134.0$. EIMS: $\mathrm{m} / z$ (rel intensity) 203 (M+-Br, 0.2), 185 (1), 163 (8), 121 (14), 95 (14), 83 (54), 55 (100), 41 (30). Anal. calcd for $\mathrm{C}_{8} \mathrm{H}_{12} \mathrm{Br}_{2} \mathrm{O}$ : C, 33.83; H, 4.26. Found: C, 34.05; H, 4.36. 


\section{References:}

1. Mandai, T.; Nokami, J.; Yano, T.; Yoshinaga, Y.; Otera, J. J. Org Chem. 1984, 49, 172.

2. Harris, G. D., Jr.; Herr, R. J.; Weinreb, S. M. J. Org. Chem. 1993, 58, 5452.

3. Dhillon, R. S.; Singh, S.; Singh, J. Indian J. Chem. Sect. B 1994, 33, 365.

4. Luo, Y.; Herndon, J. W.; Cervantes-Lee, F. J. Am. Chem. Soc. 2003, 125, 12720.

5. Felpin, F.-X.; Bertrand, M.-J.; Lebreton, J. Tetrahedron 2002, 58, 7381.

\section{Typical procedures for $\mathrm{Cu}$-catalyzed and Pd-catalyzed O-vinylation}

\section{Typical Procedure for Copper-Catalyzed Intramolecular Coupling of Vinyl Bromides}

with Alcohols: $\gamma$-Bromohomoallylic alcohol $1 \mathrm{a}(83 \mathrm{mg}, 0.3 \mathrm{mmol})$ in dry $\mathrm{CH}_{3} \mathrm{CN}(3 \mathrm{~mL})$ was added to the mixture of $\mathrm{CuI}(5.7 \mathrm{mg}, 0.03 \mathrm{mmol}), 1,10$-phenanthroline $(10.8 \mathrm{mg}, 0.06 \mathrm{mmol})$, and $\mathrm{Cs}_{2} \mathrm{CO}_{3}(196 \mathrm{mg}, 0.6 \mathrm{mmol})$ in a round flask at room temperature under nitrogen. The mixture was then stirred at refluxing temperature for $8 \mathrm{~h}$. TLC monitoring indicated that all the starting material 1a was consumed. The resulting mixture was then cooled down to room temperature, and ethyl ether $(20 \mathrm{~mL})$ was added. The mixture was filtered and the filtrate was concentrated under reduced pressure. The crude product was then purified by column chromatography on basic alumina with hexane/ethyl ether/triethylamine $(10: 1: 0.1, \mathrm{v} / \mathrm{v} / \mathrm{v})$ as the eluent to give the 2-methyleneoxetane 2a as a colorless oil. Yield: $57.6 \mathrm{mg}(98 \%)$.

\section{Typical Procedure for Palladium-Catalyzed Intramolecular O-Vinylation: Alcohol 1q (81} $\mathrm{mg}, 0.3 \mathrm{mmol})$ in dry THF $(3 \mathrm{~mL})$ was added to the mixture of $\mathrm{Pd}(\mathrm{OAc})_{2}(3.4 \mathrm{mg}, 0.015 \mathrm{mmol})$, BINAP (14 mg, $0.023 \mathrm{mmol})$, and $\mathrm{Cs}_{2} \mathrm{CO}_{3}(196 \mathrm{mg}, 0.6 \mathrm{mmol})$ in a round flask at room temperature under nitrogen. The mixture was stirred at refluxing temperature for $40 \mathrm{~h}$. TLC monitoring indicated that all the starting material 1q was consumed. The resulting mixture was then cooled to room temperature and ethyl ether $(20 \mathrm{~mL})$ was added. The mixture was filtered and the filtrate was concentrated under reduced pressure. The crude product was then purified by column chromatography on basic alumina with hexane/ethyl ether/triethylamine (10:1:0.1, $\mathrm{v} / \mathrm{v} / \mathrm{v})$ as the eluent to give the $\mathbf{2 q}$ as a colorless oil. Yield: $53 \mathrm{mg}(94 \%)$. 


\section{Characterizations of products $2,6,8$ and 9}

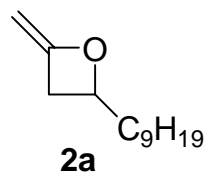

Colorless oil. IR (film): $v\left(\mathrm{~cm}^{-1}\right)$ 2927, 2855, 1691, 1465, 1190, 1083, 956, 782. ${ }^{1} \mathrm{H}$ NMR (300 $\left.\mathrm{MHz}, \mathrm{CDCl}_{3}\right) \delta 0.81(3 \mathrm{H}, \mathrm{t}, J=6.9 \mathrm{~Hz}), 1.19(14 \mathrm{H}, \mathrm{s}), 1.53-1.65(1 \mathrm{H}, \mathrm{m}), 1.74-1.81(1 \mathrm{H}, \mathrm{m})$, 2.69-2.76 (1H, m), 3.12-3.20 (1H, m), $3.66(1 \mathrm{H}, \mathrm{t}, J=1.5 \mathrm{~Hz}), 4.02-4.04(1 \mathrm{H}, \mathrm{m}), 4.63-4.72$ $(1 \mathrm{H}, \mathrm{m}) .{ }^{13} \mathrm{C}$ NMR $\left(75.4 \mathrm{MHz}, \mathrm{CDCl}_{3}\right) \delta 14.1,22.7,24.3,29.3,29.4,29.48,29.53,31.9,34.1$, 36.1, 79.2, 79.6, 162.6. EIMS: $m / z$ (rel intensity) $138\left(\mathrm{M}^{+}-58,2\right), 121$ (1), 110 (3), 97 (16), 81 (11), 69 (26), 55 (65), 43 (100). Anal. calcd for $\mathrm{C}_{13} \mathrm{H}_{24} \mathrm{O}$ : C, 79.53; H, 12.32. Found: C, 79.50; $\mathrm{H}, 12.36$.

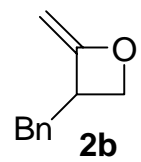

Colorless oil. IR (film): $v\left(\mathrm{~cm}^{-1}\right)$ 3028, 2895, 1687, 1496, 1454, 1185, 954, 890, 792, 699. ${ }^{1} \mathrm{H}$ NMR $\left(300 \mathrm{MHz}, \mathrm{CDCl}_{3}\right) \delta 2.96(2 \mathrm{H}, \mathrm{d}, J=8.4 \mathrm{~Hz}), 3.60(1 \mathrm{H}, \mathrm{q}, J=1.8 \mathrm{~Hz}), 3.65-3.70(1 \mathrm{H}$, m), $4.04\left(1 \mathrm{H}, \mathrm{dd}, J_{1}=2.4 \mathrm{~Hz}, J_{2}=3.6 \mathrm{~Hz}\right), 4.34(1 \mathrm{H}, \mathrm{t}, J=5.4 \mathrm{~Hz}), 4.58-4.62(1 \mathrm{H}, \mathrm{m})$, 7.09-7.26 (5H, m). ${ }^{13} \mathrm{C}$ NMR (75.4 MHz, $\left.\mathrm{CDCl}_{3}\right) \delta 38.3,42.8,73.9,79.3,126.5,128.5,128.6$, 138.4, 168.9. EIMS: m/z (rel intensity) $160\left(\mathbf{M}^{+}, 4\right), 147$ (14), 129 (11), 117 (34), 91 (100), 83 (17), 55 (41), 43 (84). HRMS calcd for $\mathrm{C}_{11} \mathrm{H}_{12} \mathrm{O}$ : 160.0888. Found: 160.0889.

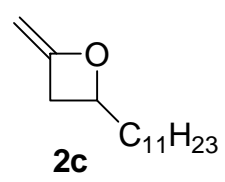

Colorless oil. IR (film): $v\left(\mathrm{~cm}^{-1}\right)$ 2925, 2854, 1693, 1240, 1189, 981, 955, 782. ${ }^{1} \mathrm{H}$ NMR (300 $\left.\mathrm{MHz}, \mathrm{CDCl}_{3}\right) \delta 0.88(3 \mathrm{H}, \mathrm{t}, J=6.6 \mathrm{~Hz}), 1.39(18 \mathrm{H}, \mathrm{s}), 1.65-1.72(1 \mathrm{H}, \mathrm{m}), 1.81-1.88(1 \mathrm{H}, \mathrm{m})$, 2.76-2.84 (1H, m), 3.20-3.27 (1H, m), 3.73-3.74 (1H, m), 4.09-4.12 (1H, m), 4.73-4.77 (1H, m). ${ }^{13} \mathrm{C} \mathrm{NMR}\left(75.4 \mathrm{MHz}, \mathrm{CDCl}_{3}\right) \delta 14.1,22.7,24.3,29.3,29.4,29.5,29.6,31.9,34.1,36.1$, 79.2, 79.6, 162.6. EIMS: m/z (rel intensity) $166\left(\mathbf{M}^{+}-58,1\right), 135$ (1), 111 (5), 97 (19), 83 (32), 
69 (27), 55 (56), 43 (100). Anal. calcd for $\mathrm{C}_{15} \mathrm{H}_{28} \mathrm{O}: \mathrm{C}, 80.29 ; \mathrm{H}, 12.58$. Found: C, 79.99; H, 12.30 .

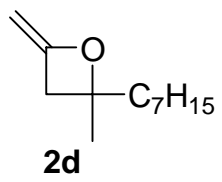

Colorless oil. IR (film): $v\left(\mathrm{~cm}^{-1}\right)$ 2929, 2857, 1695, 1464, 1378, 948, 780. ${ }^{1} \mathrm{H}$ NMR $(300 \mathrm{MHz}$, $\left.\mathrm{CDCl}_{3}\right) \delta$ 0.79-0.83 (3H, m), 1.21-1.30 (10H, m), $1.39(3 \mathrm{H}, \mathrm{s}), 1.64-1.67(2 \mathrm{H}, \mathrm{m}), 2.82(2 \mathrm{H}, \mathrm{dd}$, $\left.J_{1}=14.7 \mathrm{~Hz}, J_{2}=33.0 \mathrm{~Hz}\right), 3.67-3.68(1 \mathrm{H}, \mathrm{m}), 4.03\left(1 \mathrm{H}, \mathrm{dd}, J_{1}=2.4 \mathrm{~Hz}, J_{2}=4.5 \mathrm{~Hz}\right) .{ }^{13} \mathrm{C}$ NMR $\left(75.4 \mathrm{MHz}, \mathrm{CDCl}_{3}\right) \delta 14.1,22.6,23.7,25.3,29.2,29.8,31.7,39.2,40.5,79.9,84.7$, 160.2. EIMS: $m / z$ (rel intensity) $182\left(\mathrm{M}^{+}, 1\right), 149$ (6), 124 (6), 97 (24), 83 (32), 69 (49), 56 (92), 43 (100). HRMS calcd for $\mathrm{C}_{12} \mathrm{H}_{22} \mathrm{O}: 182.1671$. Found: 182.1678.

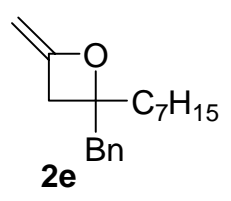

Colorless oil. IR (film): $v\left(\mathrm{~cm}^{-1}\right)$ 3029, 2929, 2856, 1693, 1604, 1496, 1455, 1429, 953, 909 , 785, 747, 700. ${ }^{1} \mathrm{H}$ NMR (300 MHz, $\left.\mathrm{CDCl}_{3}\right) \delta 0.79-0.84(3 \mathrm{H}, \mathrm{m}), 1.21(8 \mathrm{H}, \mathrm{d}, J=2.7 \mathrm{~Hz})$, 1.31-1.39 (2H, m), 1.59-1.64 (2H, m), 2.77-2.84 (2H, m), $2.96\left(2 \mathrm{H}, \mathrm{dd}, J_{1}=13.8 \mathrm{~Hz}, J_{2}=20.1\right.$ $\mathrm{Hz}), 3.61-3.63(1 \mathrm{H}, \mathrm{m}), 4.03\left(1 \mathrm{H}, \mathrm{dd}, J_{1}=2.4 \mathrm{~Hz}, J_{2}=5.4 \mathrm{~Hz}\right), 7.16-7.26(5 \mathrm{H}, \mathrm{m}) .{ }^{13} \mathrm{C} \mathrm{NMR}$ (75.4 MHz, $\left.\mathrm{CDCl}_{3}\right) \delta 14.1,22.6,23.3,29.1,29.7,31.7,37.0,37.8,43.9,79.8,86.2,126.6$, 128.2, 130.0, 136.3, 160.1. EIMS: $m / z$ (rel intensity) $258\left(\mathrm{M}^{+}, 8\right), 243$ (5), 200 (42), 159 (29), 131 (55), 117 (100), 91 (84), 43 (86). HRMS calcd for $\mathrm{C}_{18} \mathrm{H}_{26} \mathrm{O}: 258.1984$. Found: 258.1982.

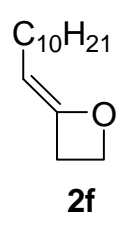

Colorless oil. IR (film): $v\left(\mathrm{~cm}^{-1}\right)$ 2924, 2853, 1717, 1465, 1320, 948, 883, 794. ${ }^{1} \mathrm{H}$ NMR (300 $\left.\mathrm{MHz}, \mathrm{CDCl}_{3}\right) \delta 0.81(3 \mathrm{H}, \mathrm{t}, J=4.2 \mathrm{~Hz}), 1.19-1.22(16 \mathrm{H}, \mathrm{m}), 1.85(2 \mathrm{H}, \mathrm{t}, J=3.9 \mathrm{~Hz}), 3.09-3.12$ $(2 \mathrm{H}, \mathrm{m}), 3.95(1 \mathrm{H}, \mathrm{t}, J=4.2 \mathrm{~Hz}), 4.54(2 \mathrm{H}, \mathrm{t}, J=4.2 \mathrm{~Hz}) .{ }^{13} \mathrm{C} \mathrm{NMR}\left(75.4 \mathrm{MHz}, \mathrm{CDCl}_{3}\right) \delta 14.1$, 22.7, 22.8, 28.7, 29.3, 29.4, 29.5, 29.6, 29.7, 30.2, 31.9, 68.0, 96.4, 156.5. EIMS: $m / z$ (rel intensity) $210\left(\mathrm{M}^{+}, 1\right), 166$ (2), 126 (4), 108 (4), 97 (9), 83 (100), 55 (78), 41 (34). HRMS calcd 
for $\mathrm{C}_{14} \mathrm{H}_{26} \mathrm{O}: 210.1984$. Found: 210.1994 . The stereochemistry of $\mathbf{2 f}$ was confirmed by its NOESY experiments.

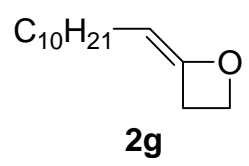

Colorless oil. IR (film): $v\left(\mathrm{~cm}^{-1}\right)$ 2924, 2853, 1715, 1465, 1261, 1088, 988, 935, 802. ${ }^{1} \mathrm{H}$ NMR $\left(300 \mathrm{MHz}, \mathrm{CDCl}_{3}\right) \delta 0.88(3 \mathrm{H}, \mathrm{t}, J=4.2 \mathrm{~Hz}), 1.26-1.31(16 \mathrm{H}, \mathrm{m}), 1.75-1.79(2 \mathrm{H}, \mathrm{m})$, 3.17-3.19 (2H, m), 4.53-4.56 (1H, m), $4.60(2 \mathrm{H}, \mathrm{t}, J=4.2 \mathrm{~Hz}) .{ }^{13} \mathrm{C} \mathrm{NMR}\left(75.4 \mathrm{MHz}, \mathrm{CDCl}_{3}\right) \delta$ 14.1, 22.7, 24.7, 27.8, 29.1, 29.4, 29.5, 29.65, 29.68, 30.5, 31.9, 68.0, 95.8, 157.3. EIMS: $\mathrm{m} / \mathrm{z}$ (rel intensity) $210\left(\mathrm{M}^{+}, 1\right), 193$ (1), 167 (3), 123 (6), 97 (25), 83 (64), 55 (100), 43 (99). HRMS calcd for $\mathrm{C}_{14} \mathrm{H}_{26} \mathrm{O}: 210.1984$. Found: 210.1979 .

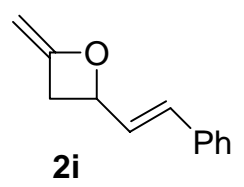

Colorless oil. IR (film): $v\left(\mathrm{~cm}^{-1}\right)$ 3027, 2947, 1691, 1494, 1450, 1268, 1185, 1151, 967, 916, 788, 748, 692. ${ }^{1} \mathrm{H}$ NMR (300 MHz, $\left.\mathrm{CDCl}_{3}\right)$ $\delta$ 2.96-3.03 (1H, m), 3.35-3.42 (1H, m), 3.73-3.76 $(1 \mathrm{H}, \mathrm{m}), 4.11-4.14(1 \mathrm{H}, \mathrm{m}), 5.25\left(1 \mathrm{H}, \mathrm{dd}, J_{1}=7.2 \mathrm{~Hz}, J_{2}=12.6 \mathrm{~Hz}\right), 6.36\left(1 \mathrm{H}, \mathrm{dd}, J_{1}=7.2 \mathrm{~Hz}\right.$, $\left.J_{2}=15.6 \mathrm{~Hz}\right), 6.60(1 \mathrm{H}, \mathrm{d}, J=15.6 \mathrm{~Hz}), 7.17-7.28(3 \mathrm{H}, \mathrm{m}), 7.34(1 \mathrm{H}, \mathrm{d}, J=8.1 \mathrm{~Hz}) .{ }^{13} \mathrm{C} \mathrm{NMR}$ $\left(75.4 \mathrm{MHz}, \mathrm{CDCl}_{3}\right) \delta 35.6,78.7,80.2,126.8,127.7,128.3,128.6,133.2$, 135.8, 162.0. EIMS: m/z (rel intensity) $172\left(\mathrm{M}^{+}, 7\right), 144$ (7), 130 (100), 115 (57), 91 (9), 77 (22), 51 (25), 43 (11). HRMS calcd for $\mathrm{C}_{12} \mathrm{H}_{12} \mathrm{O}$ : 172.0888. Found: 172.0886 .

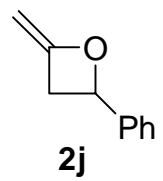

Colorless oil. IR (film): $v\left(\mathrm{~cm}^{-1}\right)$ 3032, 2950, 1694, 1456, 1269, 1183, 946, 757, 697. ${ }^{1} \mathrm{H}$ NMR

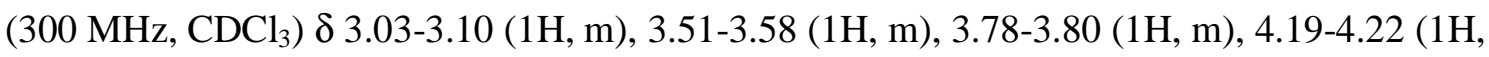
m), $5.65(1 \mathrm{H}, \mathrm{t}, J=6.0 \mathrm{~Hz}), 7.26-7.38(5 \mathrm{H}, \mathrm{m}) .{ }^{13} \mathrm{C} \mathrm{NMR}\left(75.4 \mathrm{MHz}, \mathrm{CDCl}_{3}\right) \delta 37.4,79.0$, 80.4, 125.5, 128.5, 128.6, 140.6, 162.1. EIMS: $m / z$ (rel intensity) $146\left(\mathrm{M}^{+}, 1\right), 129$ (5), 104 (100), 95 (3), 83 (15), 71 (18), 55 (29), 43 (70). HRMS calcd for $\mathrm{C}_{10} \mathrm{H}_{10} \mathrm{O}:$ 146.0732. Found: 146.0726 . 


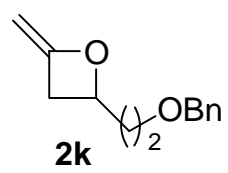

Colorless oil. IR (film): $v\left(\mathrm{~cm}^{-1}\right)$ 3028, 2954, 2860, 1691, 1494, 1363, 1189, 1102, 1082, 735, 697. ${ }^{1} \mathrm{H}$ NMR $\left(300 \mathrm{MHz}, \mathrm{CDCl}_{3}\right) \delta$ 1.93-2.10 (2H, m), 2.78-2.86 (1H, m), 3.15-3.24 (1H, m), 3.49-3.53 (2H, m), $3.68(1 \mathrm{H}, \mathrm{t}, J=1.5 \mathrm{~Hz}), 4.03-4.06(1 \mathrm{H}, \mathrm{m}), 4.42(2 \mathrm{H}, \mathrm{s}), 4.83-4.90(1 \mathrm{H}, \mathrm{m})$, 7.18-7.30 (5H, m). ${ }^{13} \mathrm{C}$ NMR (75.4 MHz, $\left.\mathrm{CDCl}_{3}\right) \delta 34.2,36.2,65.8,73.1,76.6,79.9,127.56$, 127.60, 128.4, 138.2, 162.5. EIMS: $m / z$ (rel intensity) $146\left(\mathbf{M}^{+}-58,8\right), 132$ (1), 105 (6), 91 (100), 77 (3), 65 (9), 55 (5), 43 (17). Anal. calcd for $\mathrm{C}_{13} \mathrm{H}_{16} \mathrm{O}_{2}$ : C, 76.44; H, 7.90. Found: C, $76.18 ; \mathrm{H}, 7.86$.

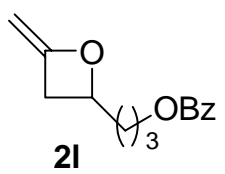

Colorless oil. IR (film): $v\left(\mathrm{~cm}^{-1}\right)$ 3063, 2949, 1719, 1690, 1602, 1452, 1275, 1114, 1070, 1027, 952, 789, 712. ${ }^{1} \mathrm{H}$ NMR $\left(300 \mathrm{MHz}, \mathrm{CDCl}_{3}\right) \delta 1.88-2.02(4 \mathrm{H}, \mathrm{m}), 2.85\left(1 \mathrm{H}, \mathrm{dd}, J_{1}=7.8 \mathrm{~Hz}, J_{2}=\right.$ $15.0 \mathrm{~Hz}), 3.28\left(1 \mathrm{H}, \mathrm{dd}, J_{1}=6.3 \mathrm{~Hz}, J_{2}=14.7 \mathrm{~Hz}\right), 3.77(1 \mathrm{H}, \mathrm{s}), 4.14(1 \mathrm{H}, \mathrm{s}), 4.37-4.39(2 \mathrm{H}, \mathrm{m})$, 4.82-4.83 (1H, m), $7.45(2 \mathrm{H}, \mathrm{t}, J=7.2 \mathrm{~Hz}), 7.54-7.59(1 \mathrm{H}, \mathrm{m}), 8.04(2 \mathrm{H}, \mathrm{d}, J=7.2 \mathrm{~Hz}) .{ }^{13} \mathrm{C}$ NMR (75.4 MHz, $\left.\mathrm{CDCl}_{3}\right) \delta 23.8,32.6,34.0,64.3,78.4,80.0,128.3,129.5,130.1,132.9,162.1$, 166.4. EIMS: $m / z$ (rel intensity) $232\left(\mathrm{M}^{+}, 1\right), 149$ (1), 123 (3), 105 (100), 95 (14), 77 (48), 68 (29), 41 (17). Anal. calcd for $\mathrm{C}_{14} \mathrm{H}_{16} \mathrm{O}_{3}$ : C, 72.39; H, 6.94. Found: C, 72.20; H, 6.97.

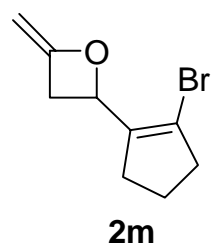

Colorless oil. IR (film): $v\left(\mathrm{~cm}^{-1}\right) 2952,2852,1693,1648,1432,1269,1181,1080,947,914 .{ }^{1} \mathrm{H}$ NMR (300 MHz, $\left.\mathrm{CDCl}_{3}\right) \delta$ 1.89-1.99 (2H, m), 2.42-2.64 (4H, m), 2.97-3.05 $(1 \mathrm{H}, \mathrm{m})$, 3.29-3.42 (1H, m), 3.70-3.72 (1H, m), 4.06-4.09 (1H, m), $5.53(1 \mathrm{H}, \mathrm{t}, J=6.3 \mathrm{~Hz}) .{ }^{13} \mathrm{C} \mathrm{NMR}$ $\left(75.4 \mathrm{MHz}, \mathrm{CDCl}_{3}\right) \delta 21.6,29.6,33.3,40.7,75.1,80.0,120.5,138.9,162.3$. EIMS: $m / z$ (rel intensity) 214 ( $\left.\mathrm{M}^{+}, 2\right), 172$ (11), 151 (18), 135 (32), 119 (7), 91 (40), 77 (28), 43 (100). HRMS calcd for $\mathrm{C}_{9} \mathrm{H}_{11} \mathrm{BrO}: 213.9993$. Found: 213.9992 . 


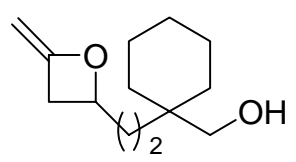

$2 n$

Colorless oil. ${ }^{1} \mathrm{H}$ NMR (300 MHz, $\left.\mathrm{CDCl}_{3}\right) \delta$ 1.19-1.36 (13H, m), 1.53-1.78 (2H, m), 2.71-2.77 $(1 \mathrm{H}, \mathrm{m}), 3.17\left(1 \mathrm{H}, \mathrm{dd}, J_{1}=6.3 \mathrm{~Hz}, J_{2}=14.4 \mathrm{~Hz}\right), 3.36(2 \mathrm{H}, \mathrm{d}, J=3.3 \mathrm{~Hz}), 3.68(1 \mathrm{H}, \mathrm{d}, J=1.8$ $\mathrm{Hz}), 4.05(1 \mathrm{H}, \mathrm{d}, J=2.1 \mathrm{~Hz}), 4.61-4.69(1 \mathrm{H}, \mathrm{m}) .{ }^{13} \mathrm{C} \mathrm{NMR}\left(75.4 \mathrm{MHz}, \mathrm{CDCl}_{3}\right) \delta 21.3,21.4$, 26.3, 28.6, 29.7, 32.3, 32.4, 34.0, 36.6, 68.0, 79.7, 79.9, 162.4. ESIMS: $m / z 233.0\left(\mathrm{M}^{+}+\mathrm{Na}\right)$. HRMS calcd for $\mathrm{C}_{13} \mathrm{H}_{22} \mathrm{O}_{2} \mathrm{Na}\left(\mathrm{M}^{+}+\mathrm{Na}\right): 233.1509$. Found: 233.1512 .

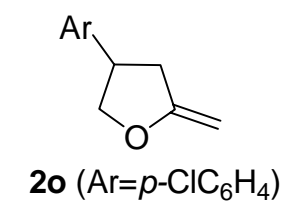

Colorless oil. IR (film): $v\left(\mathrm{~cm}^{-1}\right)$ 2953, 2884, 1672, 1598, 1495, 1224, 1093, 1039, 1014, 826, 802. ${ }^{1} \mathrm{H}$ NMR $\left(300 \mathrm{MHz}, \mathrm{CDCl}_{3}\right) \delta 2.55\left(1 \mathrm{H}, \mathrm{dd}, J_{1}=7.5 \mathrm{~Hz}, J_{2}=15.6 \mathrm{~Hz}\right), 2.88\left(1 \mathrm{H}, \mathrm{dd}, J_{1}=\right.$ $\left.7.5 \mathrm{~Hz}, J_{2}=15.6 \mathrm{~Hz}\right), 3.37-3.47(1 \mathrm{H}, \mathrm{m}), 3.85(1 \mathrm{H}, \mathrm{d}, J=1.8 \mathrm{~Hz}), 3.92-3.97(1 \mathrm{H}, \mathrm{m}), 4.22(1 \mathrm{H}$, $\mathrm{t}, J=1.5 \mathrm{~Hz}), 4.31-4.36(1 \mathrm{H}, \mathrm{m}), 7.09(2 \mathrm{H}, \mathrm{d}, J=8.1 \mathrm{~Hz}), 7.21(2 \mathrm{H}, \mathrm{d}, J=8.1 \mathrm{~Hz}) .{ }^{13} \mathrm{C} \mathrm{NMR}$ $\left(75.4 \mathrm{MHz}, \mathrm{CDCl}_{3}\right) \delta 37.0,43.0,76.1,80.3,128.3,128.8,132.7,139.6,162.1$. EIMS: $\mathrm{m} / z$ (rel intensity) 194 ( $\left.\mathrm{M}^{+}, 47\right), 152$ (29), 138 (9), 127 (20), 117 (79), 89 (10), 63 (13), 43 (100). Anal. calcd for $\mathrm{C}_{11} \mathrm{H}_{11} \mathrm{ClO}$ : C, 67.87; H, 5.70. Found: C, 67.67; H, 5.72.

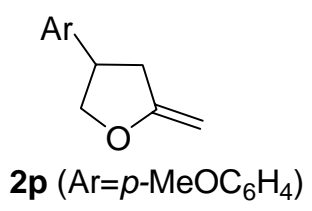

Colorless oil. IR (film): $v\left(\mathrm{~cm}^{-1}\right)$ 2954, 2836, 1672, 1612, 1515, 1295, 1250, 1181, 1037, 829, 800. ${ }^{1} \mathrm{H}$ NMR $\left(300 \mathrm{MHz}, \mathrm{CDCl}_{3}\right) \delta 2.64\left(1 \mathrm{H}, \mathrm{dd}, J_{1}=8.1 \mathrm{~Hz}, J_{2}=15.9 \mathrm{~Hz}\right), 2.92\left(1 \mathrm{H}, \mathrm{dd}, J_{1}=\right.$ $\left.8.1 \mathrm{~Hz}, J_{2}=15.3 \mathrm{~Hz}\right), 3.44-3.49(1 \mathrm{H}, \mathrm{m}), 3.78(3 \mathrm{H}, \mathrm{s}), 3.90(1 \mathrm{H}, \mathrm{s}), 3.99(1 \mathrm{H}, \mathrm{t}, J=8.1 \mathrm{~Hz})$, $4.27(1 \mathrm{H}, \mathrm{s}), 4.36-4.41(1 \mathrm{H}, \mathrm{m}), 6.85(2 \mathrm{H}, \mathrm{d}, J=8.4 \mathrm{~Hz}), 7.15(2 \mathrm{H}, \mathrm{d}, J=8.4 \mathrm{~Hz}) .{ }^{13} \mathrm{C} \mathrm{NMR}$ $\left(75.4 \mathrm{MHz}, \mathrm{CDCl}_{3}\right) \delta 37.2,42.9,55.2,76.4,79.8,114.0,127.9,132.8,158.5,162.6$. EIMS: $\mathrm{m} / z$ (rel intensity) $190\left(\mathrm{M}^{+}, 82\right), 159$ (12), 148 (50), 121 (49), 91 (24), 77 (17), 65 (13), 43 (100). HRMS calcd for $\mathrm{C}_{12} \mathrm{H}_{14} \mathrm{O}_{2}: 190.0994$. Found: 190.1003. 


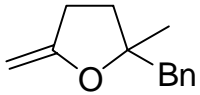

$2 q$

Colorless oil. IR (film): v ( $\left.\mathrm{cm}^{-1}\right)$ 3028, 2970, 2930, 1670, 1603, 1496, 1453, 1378, 1187, 1089, 988, 791, 701. ${ }^{1} \mathrm{H}$ NMR (300 MHz, $\left.\mathrm{CDCl}_{3}\right) \delta 1.29(3 \mathrm{H}, \mathrm{s}), 1.69-1.78(1 \mathrm{H}, \mathrm{m}), 1.93-2.02(1 \mathrm{H}$, m), 2.42-2.52 (1H, m), 2.56-2.65 (1H, m), $2.88(2 \mathrm{H}, \mathrm{s}), 3.78(1 \mathrm{H}, \mathrm{d}, J=1.2 \mathrm{~Hz}), 4.20(1 \mathrm{H}, \mathrm{s})$, 7.23-7.34 (5H, m). ${ }^{13} \mathrm{C}$ NMR (75.4 MHz, $\left.\mathrm{CDCl}_{3}\right) \delta$ 25.4, 29.0, 34.8, 46.2, 78.7, 86.6, 126.3, 127.9, 130.3, 137.3, 162.3. EIMS: $m / z$ (rel intensity) $188\left(\mathrm{M}^{+}, 10\right), 171(2), 145$ (5), $131(10)$, 115 (36), 97 (100), 79 (7), 43 (85). HRMS calcd for $\mathrm{C}_{13} \mathrm{H}_{16} \mathrm{O}$ : 188.1201. Found: 188.1196.

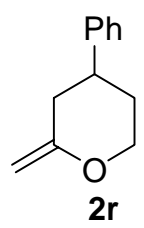

Colorless oil. IR (film): $v\left(\mathrm{~cm}^{-1}\right)$ 3029, 2948, 2867, 1652, 1494, 1453, 1254, 1081, 1040, 974, 800, 699. ${ }^{1} \mathrm{H}$ NMR (300 MHz, $\left.\mathrm{CDCl}_{3}\right) \delta$ 1.86-1.99 (2H, m), 2.31-2.40 (1H, m), $2.50(1 \mathrm{H}, \mathrm{dd}$, $\left.J_{1}=4.2 \mathrm{~Hz}, J_{2}=13.8 \mathrm{~Hz}\right), 2.82-2.91(1 \mathrm{H}, \mathrm{m}), 3.72-3.80(1 \mathrm{H}, \mathrm{m}), 4.14(1 \mathrm{H}, \mathrm{s}), 4.22-4.27(1 \mathrm{H}$, m), $4.43(1 \mathrm{H}, \mathrm{s}), 7.21-7.25(3 \mathrm{H}, \mathrm{m}), 7.31-7.36(2 \mathrm{H}, \mathrm{m}) .{ }^{13} \mathrm{C} \mathrm{NMR}\left(75.4 \mathrm{MHz}, \mathrm{CDCl}_{3}\right) \delta 32.5$, 36.9, 40.9, 69.1, 92.2, 126.5, 126.6, 128.6, 144.9, 159.4. EIMS: $m / z$ (rel intensity) $174\left(\mathrm{M}^{+}, 72\right)$, 159 (65), 131 (61), 117 (100), 104 (69), 91 (49), 77 (42), 43 (98). HRMS calcd for $\mathrm{C}_{12} \mathrm{H}_{14} \mathrm{O}$ : 174.1045. Found: 174.1046.

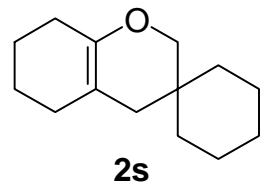

Colorless oil. IR (film): $v\left(\mathrm{~cm}^{-1}\right)$ 2926, 2858, 1697, 1449, 1260, 1203, 1156, 1065, 1026, 804. ${ }^{1} \mathrm{H}$ NMR $\left(300 \mathrm{MHz}, \mathrm{CDCl}_{3}\right) \delta 1.19-1.30(10 \mathrm{H}, \mathrm{m}), 1.40-1.62(6 \mathrm{H}, \mathrm{m}), 1.80(2 \mathrm{H}, \mathrm{s}), 1.90(2 \mathrm{H}$, s), $3.50(2 \mathrm{H}, \mathrm{s}) .{ }^{13} \mathrm{C} \mathrm{NMR}\left(75.4 \mathrm{MHz}, \mathrm{CDCl}_{3}\right) \delta$ 21.7, 23.0, 23.2, 26.6, 26.8, 29.0, 31.8, 33.7, 37.3, 73.6, 103.0, 145.2. EIMS: $m / z$ (rel intensity) $206\left(\mathrm{M}^{+}, 32\right), 177$ (4), 149 (8), 111 (100), 81 (69), 67 (65), 55 (62), 41 (66). HRMS calcd for $\mathrm{C}_{14} \mathrm{H}_{22} \mathrm{O}$ : 206.1671. Found: 206.1678. 


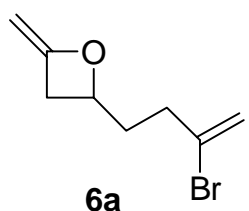

Colorless oil. IR (film): $v\left(\mathrm{~cm}^{-1}\right)$ 2949, 1693, 1434, 1260, 1240, 1188, 1125, 956, 794. ${ }^{1} \mathrm{H}$ NMR $\left(300 \mathrm{MHz}, \mathrm{CDCl}_{3}\right) \delta$ 2.01-2.06 (2H, m), 2.50-2.52 (2H, m), 2.83-2.87 (1H, m), 3.25-3.29 $(1 \mathrm{H}$, m), 3.76-3.77 $(1 \mathrm{H}, \mathrm{m}), 4.12-4.13(1 \mathrm{H}, \mathrm{m}), 4.75-4.80(1 \mathrm{H}, \mathrm{m}), 5.43(1 \mathrm{H}, \mathrm{d}, J=1.2 \mathrm{~Hz}), 5.63$ $(1 \mathrm{H}, \mathrm{s}) .{ }^{13} \mathrm{C}$ NMR $\left(75.4 \mathrm{MHz}, \mathrm{CDCl}_{3}\right) \delta 34.0,34.4,36.5,77.5,80.2,117.5,133.0,162.1$. EIMS: m/z (rel intensity) $202\left(\mathrm{M}^{+}, 1\right), 161$ (3), 123 (29), 95 (16), 81 (100), 65 (17), 55 (50), 41 (85). HRMS. calcd for $\mathrm{C}_{8} \mathrm{H}_{11} \mathrm{BrO}$ : 201.9993. Found: 201.9998. The structure of compound 6a was further confirmed by its COSY experiments.

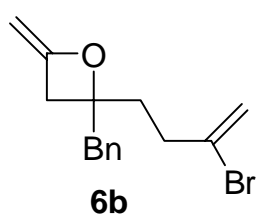

Colorless oil. IR (film): $v\left(\mathrm{~cm}^{-1}\right)$ 3028, 2945, 1690, 1630, 1496, 1454, 1430, 1227, 1197, 1078, 949, 906, 789, 745, 701. ${ }^{1} \mathrm{H} \mathrm{NMR}\left(300 \mathrm{MHz}, \mathrm{CDCl}_{3}\right) \delta 1.90\left(2 \mathrm{H}, \mathrm{dd}, J_{1}=7.2 \mathrm{~Hz}, J_{2}=15.0 \mathrm{~Hz}\right)$, $2.54(2 \mathrm{H}, \mathrm{t}, J=7.8 \mathrm{~Hz}), 2.87(2 \mathrm{H}, \mathrm{d}, 6.9 \mathrm{~Hz}), 2.97(2 \mathrm{H}, \mathrm{s}), 3.64(1 \mathrm{H}, \mathrm{s}), 4.04(1 \mathrm{H}, \mathrm{s}), 5.32(1 \mathrm{H}$, s), $5.52(1 \mathrm{H}, \mathrm{s}), 7.19-7.23(5 \mathrm{H}, \mathrm{m}) .{ }^{13} \mathrm{C} \mathrm{NMR}\left(75.4 \mathrm{MHz}, \mathrm{CDCl}_{3}\right) \delta 35.5,36.4,36.9,44.3,80.4$, 85.1, 117.0, 126.8, 128.4, 130.0, 133.5, 135.8, 159.4. EIMS: $m / z$ (rel intensity) $292\left(\mathbf{M}^{+}, 8\right)$, 234 (5), 213 (38), 173 (30), 155 (78), 129 (49), 91 (100), 43 (46). HRMS calcd for $\mathrm{C}_{15} \mathrm{H}_{17} \mathrm{BrO}$ : 292.0463. Found: 292.0455. The structure of compound $\mathbf{6 b}$ was further confirmed by its COSY and NOESY experiments.

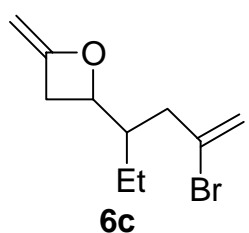

Two stereoisomers in a 2:1 ratio determined by ${ }^{1} \mathrm{H}$ NMR (300 MHz). Colorless oil. IR (film): v $\left(\mathrm{cm}^{-1}\right)$ 2964, 2933, 2878, 1692, 1629, 1462, 1431, 1191, 956, 888, 787. ${ }^{1} \mathrm{H}$ NMR (300 MHz, $\left.\mathrm{CDCl}_{3}\right) \delta$ 0.92-0.98 $(3 \mathrm{H}, \mathrm{m}), 1.38-1.55(2 \mathrm{H}, \mathrm{m}), 2.05-2.16(1 \mathrm{H}, \mathrm{m}), 2.40\left(1 \mathrm{H}, \mathrm{dd}, J_{1}=8.4 \mathrm{~Hz}\right.$, $\left.J_{2}=15.0 \mathrm{~Hz}\right), 2.63-2.70(1 \mathrm{H}, \mathrm{m}), 2.93-3.00(1 \mathrm{H}, \mathrm{m}), 3.15-3.25(1 \mathrm{H}, \mathrm{m}), 3.75-3.76(1 \mathrm{H}, \mathrm{m})$, 
4.12-4.13 (1H, m), 4.69-4.76 (1H, m), 5.46-5.48 (1H, m), 5.54-5.63 (1H, m). ${ }^{13} \mathrm{C}$ NMR $(75.4$ $\left.\mathrm{MHz}, \mathrm{CDCl}_{3}\right) \delta 10.4 / 10.9,20.9 / 21.0,32.4 / 32.6,40.5,42.1 / 42.3,79.6 / 79.7,80.3 / 80.4$, 118.6/118.7, 132.4/132.5, 162.1/162.2. EIMS: $m / z$ (rel intensity) $230\left(\mathbf{M}^{+}, 4\right), 201$ (4), 151 (40), 111 (100), 93 (32), 79 (24), 67 (21), 41 (36). HRMS calcd for $\mathrm{C}_{10} \mathrm{H}_{15} \mathrm{BrO}$ : 230.0306. Found: 230.0314 .

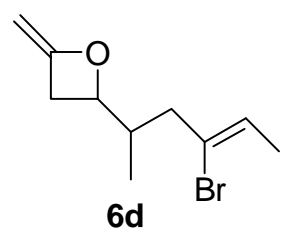

Two stereoisomers in a 3:2 ratio determined by ${ }^{1} \mathrm{H}$ NMR $(300 \mathrm{MHz})$. Colorless oil. IR (film): v $\left(\mathrm{cm}^{-1}\right)$ 2960, 2919, 1692, 1459, 1432, 1380, 1284, 1190, 958, 784. ${ }^{1} \mathrm{H}$ NMR (300 MHz, $\left.\mathrm{CDCl}_{3}\right)$ $\delta$ 0.76-1.01 (3H, m), 1.66-1.71 (3H, m), 2.04-2.68 (3H, m), 2.79-2.93 (1H, m), 3.05-3.15 (1H, m), 3.66-3.69 (1H, m), 4.03-4.06 (1H, m), 4.41-4.52 (1H, m), 5.66-5.68 (1H, m). ${ }^{13} \mathrm{C}$ NMR (75.4 MHz, $\left.\mathrm{CDCl}_{3}\right) \delta$ 12.4/13.2, 16.9, 32.0/32.1, 36.3/36.7, 43.2/43.7, 79.6/79.8, 81.7/81.8, 125.1/125.3, 126.6, 162.0/162.3. EIMS: $m / z$ (rel intensity) $230\left(\mathrm{M}^{+}, 0.5\right), 188(0.5), 151(8)$, 133 (6), 97 (100), 79 (18), 55 (61), 43 (62). HRMS calcd for $\mathrm{C}_{10} \mathrm{H}_{15} \mathrm{BrO}$ : 230.0306. Found: 230.0309 .

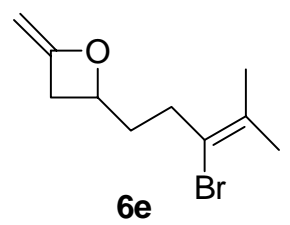

Colorless oil. IR (film): $v\left(\mathrm{~cm}^{-1}\right)$ 2920, 2857, 1692, 1434, 1191, 1011, 955, 786. ${ }^{1} \mathrm{H}$ NMR (300 $\left.\mathrm{MHz}, \mathrm{CDCl}_{3}\right) \delta 1.51(3 \mathrm{H}, \mathrm{d}, J=4.2 \mathrm{~Hz}), 1.71(3 \mathrm{H}, \mathrm{d}, J=3.3 \mathrm{~Hz}), 1.92-2.00(2 \mathrm{H}, \mathrm{m}), 2.54(2 \mathrm{H}$, d, $J=4.2 \mathrm{~Hz}), 2.79(1 \mathrm{H}, \mathrm{d}, J=14.7 \mathrm{~Hz}), 3.16-3.22(1 \mathrm{H}, \mathrm{m}), 3.68(1 \mathrm{H}, \mathrm{s}), 4.05(1 \mathrm{H}, \mathrm{d}, J=0.9$ $\mathrm{Hz}), 4.66-4.69(1 \mathrm{H}, \mathrm{m}) .{ }^{13} \mathrm{C} \mathrm{NMR}\left(75.4 \mathrm{MHz}, \mathrm{CDCl}_{3}\right) \delta 20.4,25.4,32.6,34.0,34.5,77.8,80.0$, 120.0, 131.4, 162.4. EIMS: m/z (rel intensity) $230\left(\mathrm{M}^{+}, 2\right), 190$ (1), 174 (19), 151 (57), 109 (46), 83 (60), 67 (100), 41 (48). HRMS calcd for $\mathrm{C}_{10} \mathrm{H}_{15} \mathrm{BrO}$ : 230.0306. Found: 230.0301. 


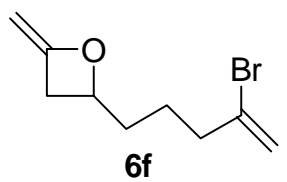

Colorless oil. IR (film): $v\left(\mathrm{~cm}^{-1}\right)$ 2943, 2864, 1690, 1630, 1432, 1188, 946, 889, 786. ${ }^{1} \mathrm{H}$ NMR $\left(300 \mathrm{MHz}, \mathrm{CDCl}_{3}\right) \delta$ 1.50-1.80 (4H, m), 2.38-2.43 (2H, m), 2.71-2.79 (1H, m), 3.15-3.22 (1H, m), 3.67-3.69 $(1 \mathrm{H}, \mathrm{m}), 4.03-4.06(1 \mathrm{H}, \mathrm{m}), 4.66-4.72(1 \mathrm{H}, \mathrm{m}), 5.35(1 \mathrm{H}, \mathrm{s}), 5.52(1 \mathrm{H}, \mathrm{s}) .{ }^{13} \mathrm{C}$ NMR (75.4 MHz, $\left.\mathrm{CDCl}_{3}\right) \delta 22.6,34.0,34.6,40.8,78.6,79.9,117.0,133.7,162.2$. EIMS: $\mathrm{m} / z$ (rel intensity) $216\left(\mathrm{M}^{+}, 2\right), 167$ (5), 149 (9), 111 (14), 97 (26), 79 (20), 55 (58), 43 (100). HRMS calcd for $\mathrm{C}_{9} \mathrm{H}_{13} \mathrm{BrO}: 216.0150$. Found: 216.0152 .

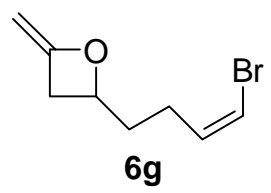

Colorless oil. IR (film): $v\left(\mathrm{~cm}^{-1}\right)$ 2942, 1691, 1433, 1290, 1188, 955, 787, 707, 669. ${ }^{1} \mathrm{H}$ NMR $\left(300 \mathrm{MHz}, \mathrm{CDCl}_{3}\right) \delta 1.78-1.89(1 \mathrm{H}, \mathrm{m}), 1.93-2.09(1 \mathrm{H}, \mathrm{m}), 2.30\left(2 \mathrm{H}, \mathrm{dd}, J_{1}=7.2 \mathrm{~Hz}, J_{2}=14.7\right.$ $\mathrm{Hz}), 2.81-2.88(1 \mathrm{H}, \mathrm{m}), 3.24-3.31(1 \mathrm{H}, \mathrm{m}), 3.76(1 \mathrm{H}, \mathrm{t}, J=1.5 \mathrm{~Hz}), 4.12-4.13(1 \mathrm{H}, \mathrm{m})$, 4.73-4.82 $(1 \mathrm{H}, \mathrm{m}), 6.12\left(1 \mathrm{H}, \mathrm{dd}, J_{1}=7.2 \mathrm{~Hz}, J_{2}=13.8 \mathrm{~Hz}\right), 6.21(1 \mathrm{H}, \mathrm{d}, J=6.9 \mathrm{~Hz}) .{ }^{13} \mathrm{C}$ NMR (75.4 MHz, $\left.\mathrm{CDCl}_{3}\right) \delta 24.9,34.0,34.3,78.3,80.1,108.9,133.2,162.2$. EIMS: $\mathrm{m} / z$ (rel intensity) $202\left(\mathrm{M}^{+}, 1\right), 185$ (5), 163 (8), 131 (14), 97 (19), 70 (33), 55 (54), 43 (100). HRMS calcd for $\mathrm{C}_{8} \mathrm{H}_{11} \mathrm{BrO}$ : 201.9993. Found: 201.9997.

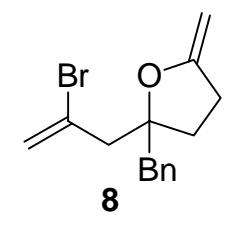

Colorless oil. ${ }^{1} \mathrm{H}$ NMR $\left(300 \mathrm{MHz}, \mathrm{CDCl}_{3}\right) \delta$ 1.79-1.89 (1H, m), 1.98-2.21 (2H, m), 2.39-2.49 $(1 \mathrm{H}, \mathrm{m}), 2.71(2 \mathrm{H}, \mathrm{s}), 2.87\left(2 \mathrm{H}, \mathrm{dd}, J_{1}=13.5 \mathrm{~Hz}, J_{2}=25.2 \mathrm{~Hz}\right), 3.65(1 \mathrm{H}, \mathrm{d}, J=1.5 \mathrm{~Hz}), 4.11$ $(1 \mathrm{H}, \mathrm{d}, J=1.2 \mathrm{~Hz}), 5.56(1 \mathrm{H}, \mathrm{d}, J=1.5 \mathrm{~Hz}), 5.65(1 \mathrm{H}, \mathrm{s}), 7.13-7.23(5 \mathrm{H}, \mathrm{m}) .{ }^{13} \mathrm{C}$ NMR $(75.4$ $\left.\mathrm{MHz}, \mathrm{CDCl}_{3}\right) \delta 29.1,32.0,44.6,49.5,79.2,87.9,121.9,126.6,127.5,128.1,130.7,136.5$, 162.2. EIMS: $m / z$ (rel intensity) $267\left(\mathrm{M}^{+}-25,10\right), 213$ (7), 171 (14), 141 (14), 123 (26), 91 (100), 73 (28), 43 (71). HRMS calcd for $\mathrm{C}_{15} \mathrm{H}_{17} \mathrm{BrO}$ : 292.0463. Found: 292.0463. 


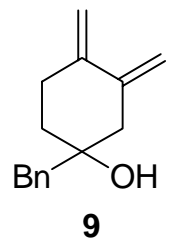

Colorless oil. IR (film): $v\left(\mathrm{~cm}^{-1}\right)$ 3449, 3079, 3028, 2936, 1637, 1495, 1453, 1438, 1088, 895, 702. ${ }^{1} \mathrm{H}$ NMR $\left(300 \mathrm{MHz}, \mathrm{CDCl}_{3}\right) \delta 1.46(1 \mathrm{H}, \mathrm{s}), 1.57-1.65(2 \mathrm{H}, \mathrm{m}), 2.15-2.38(4 \mathrm{H}, \mathrm{m}), 2.74$ $(2 \mathrm{H}, \mathrm{s}), 4.64(2 \mathrm{H}, \mathrm{s}), 4.91(1 \mathrm{H}, \mathrm{s}), 5.01(1 \mathrm{H}, \mathrm{d}, J=0.9 \mathrm{~Hz}), 7.15-7.28(5 \mathrm{H}, \mathrm{m}) .{ }^{13} \mathrm{C}$ NMR $(75.4$ $\left.\mathrm{MHz}, \mathrm{CDCl}_{3}\right) \delta 30.1,36.7,46.1,47.5,72.0,108.8,111.5,126.6,128.2,130.6,136.8,145.8$, 147.6. EIMS: $m / z$ (rel intensity) $214\left(\mathrm{M}^{+}, 4\right), 196$ (3), 165 (2), 122 (80), 95 (100), 79 (50), 55 (26), 43 (27). HRMS calcd for $\mathrm{C}_{15} \mathrm{H}_{18} \mathrm{O}: 214.1358$. Found: 214.1362.

\section{References for known compounds:}

1 Namba, K.; Kishi, Y. Org. Lett. 2004, 6, 5031.

1i

Kwon, J. S.; Pae, A. N.; Choi, K. II.; Koh, H. Y.; Kim, Y.; Cho, Y. S. Tetrahedron Lett. 2001, 42, 1957.

$\mathbf{1} \mathbf{j}$

Beruben, D.; Marek, I.; Normant, J. F.; Nicole, P. J. Org. Chem. 1995, 60, 2488

2h Dollinger, L. M.; Howell, A. R. J. Org. Chem. 1996, 61, 7248.

3 Zhang, L.-J.; Mo, X.-S.; Huang, Y.-Z. J. Organomet. Chem. 1994, 471, 77.

4 Okamoto, S.; Sato, H.; Sato, F. Tetrahedron Lett. 1996, 37, 8865.

7 Yasuda, M.; Tsuchida, M.; Baba, A. Chem. Commun. 1998, 563. 
5. COSY spectra of $6 a, 6 b$ and NOESY spectra of $2 \mathrm{f}, 6 \mathrm{~b}$.

COSY spectrum of compound $\mathbf{6 a}$
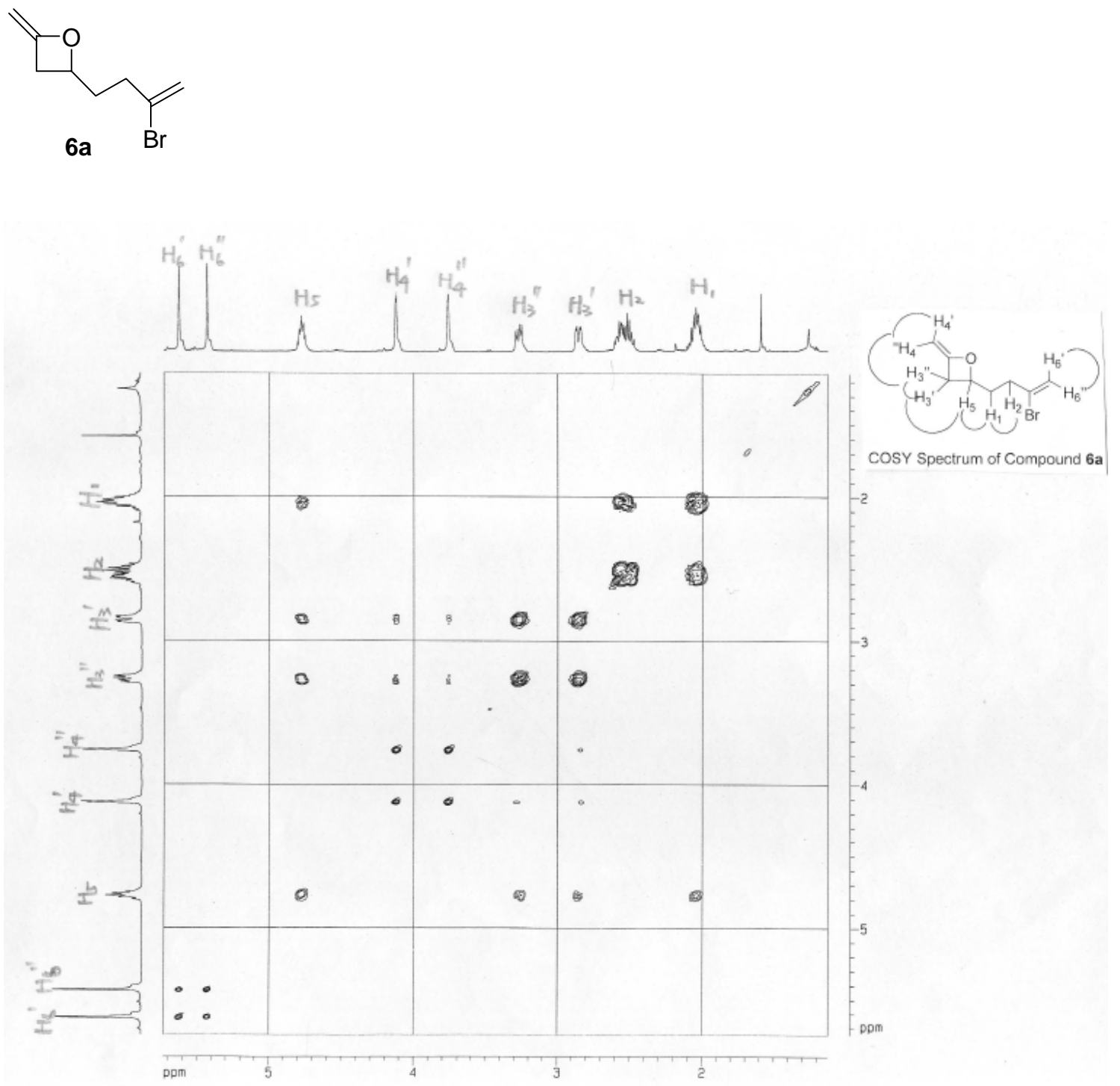
COSY spectrum of compound $\mathbf{6 b}$
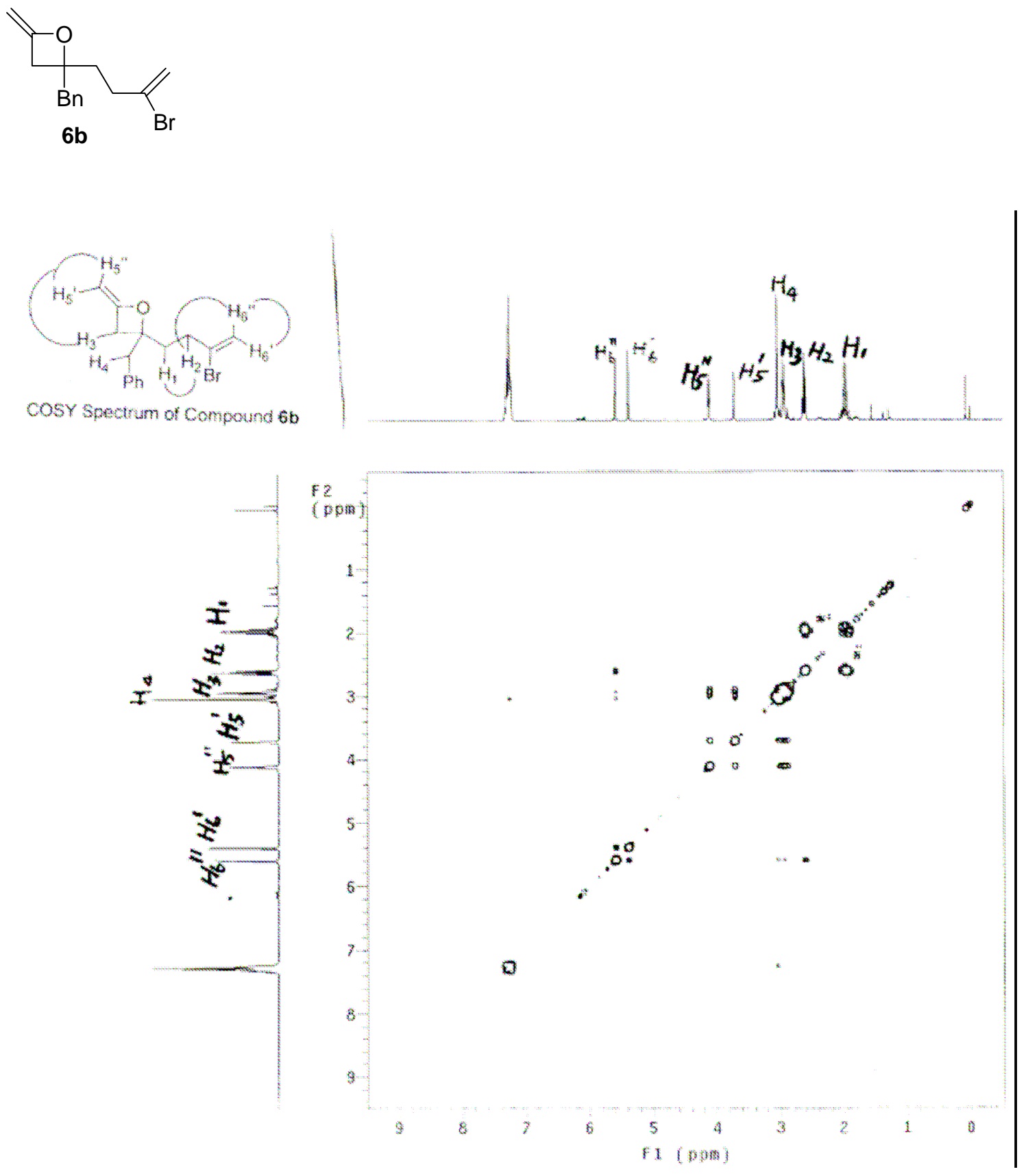


\section{NOESY spectrum of compound $\mathbf{2 f}$}
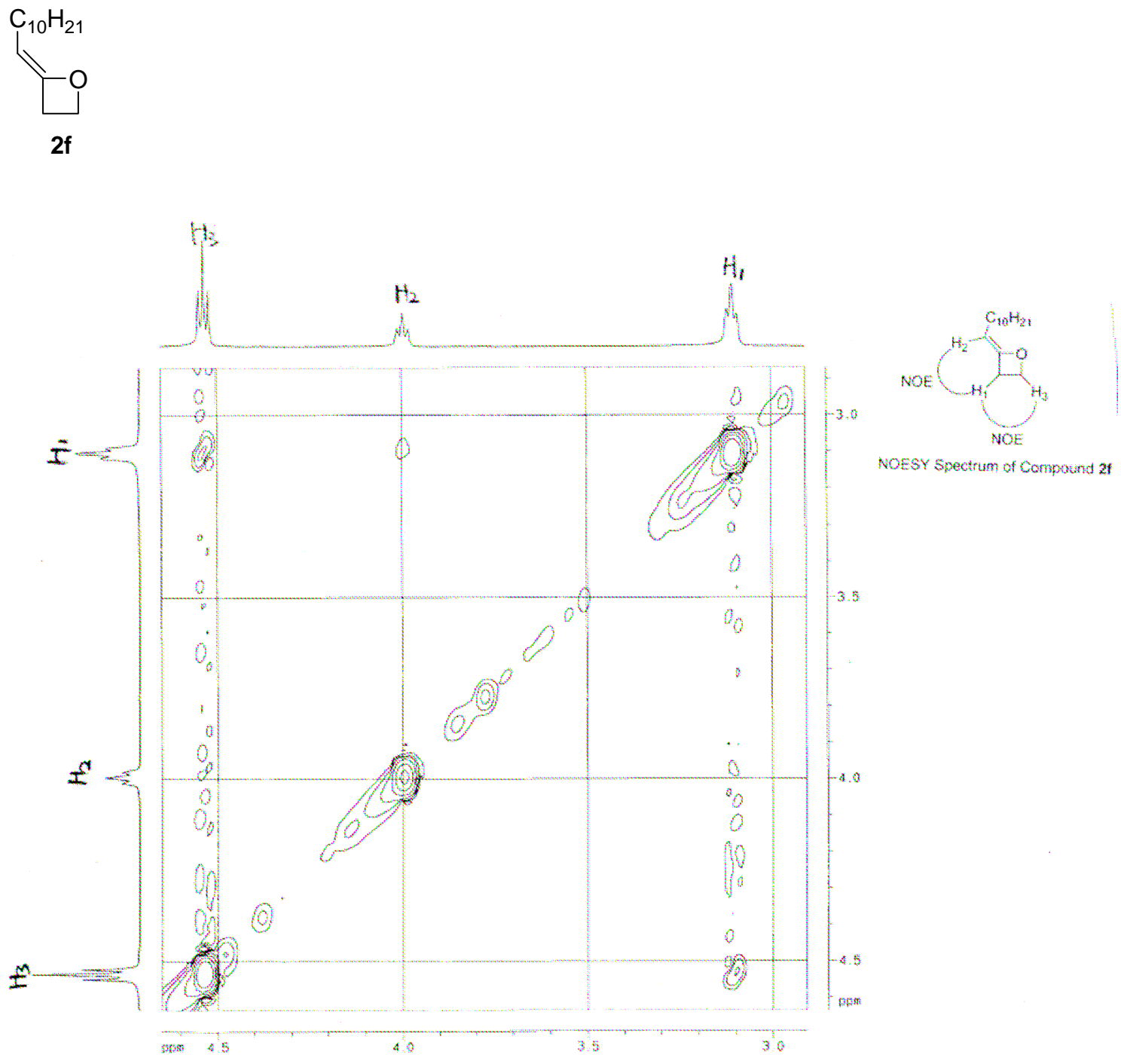


\section{NOESY spectrum of compound $\mathbf{6 b}$}
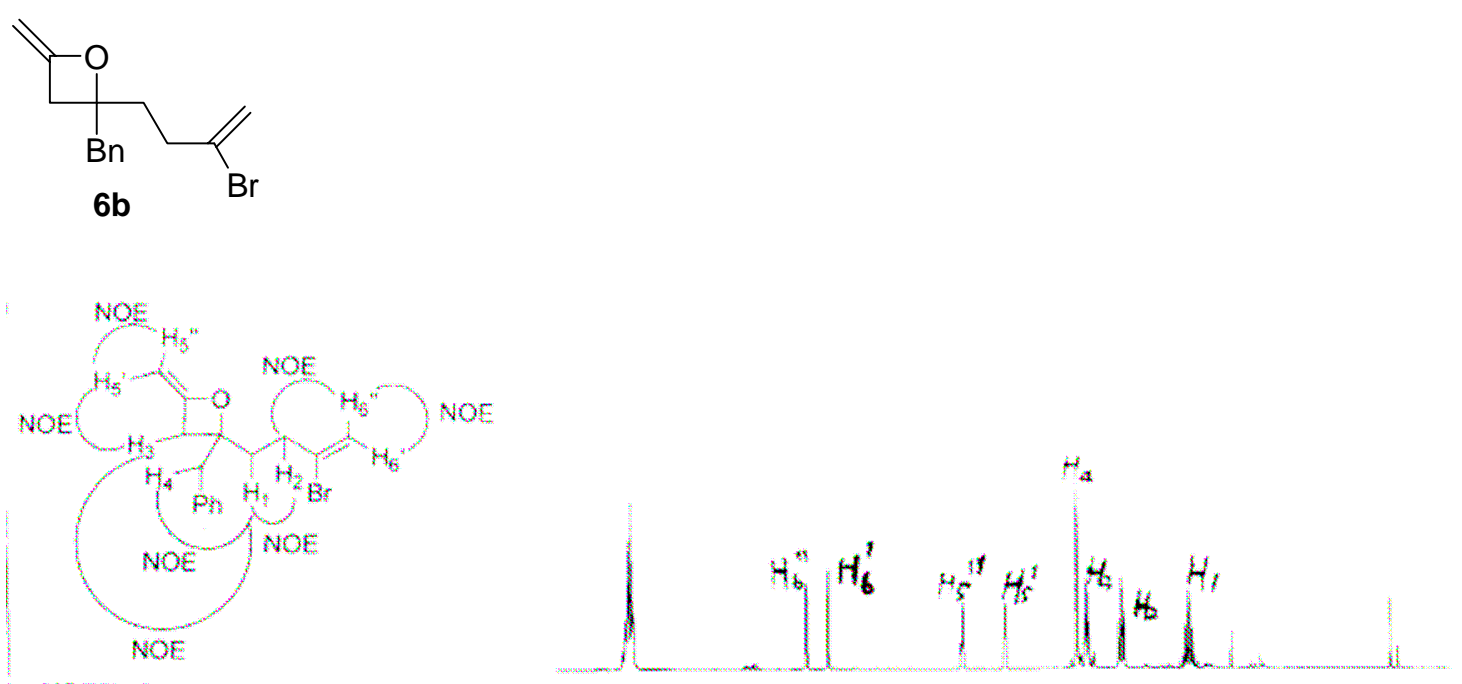

NOESY spectum of Comoound $6 \mathrm{~b}$

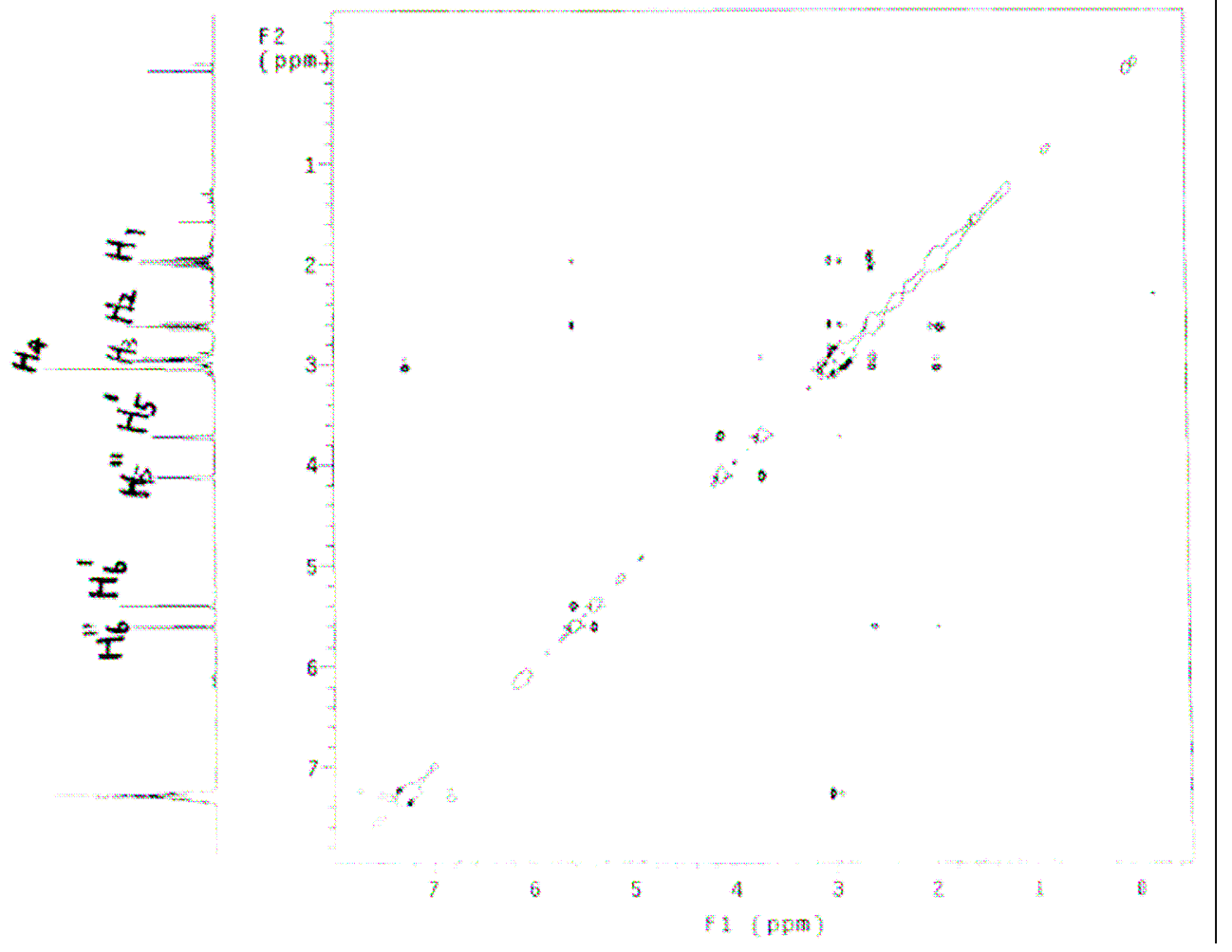

